\title{
Quelques réalisations récentes de prises en-dessous à haute altitude en Savoie
}

\section{Recently built high altitude bottom intakes}

\author{
PAR \\ X. RACT-MADOUX, M. BOUVARD, J. MOLBERT, J. ZUMSTEIN \\ RÉGION D'ÉQUIPEMENT HYDRAULIQUE ALPES II D'ÉLECTRICITÉ DE FRANCE
}

\begin{abstract}
Situation, róle, description sommaire et particularités de fonctionnement des diverses prises réalisées dans le cadre des aménagements de l'Arvan et d'Aussois (Alpes de Savoie). Présentation et commentaires des facteurs dont limportance a été révélcée ou confirmée par l'exploitation: connaissance préalable des débils liquides et solides, adaptation au sile et aménagement du lit à l'amon', caractéristiques des grilles, limitation des débits de prise, intérêt des plurges discontinues, disposition du dessableur, possibilité d'utiliser les fenêtres d'attaque comme canaux de décantation supplémentaires, efficacité des chasses et in'térêt des vannes $\dot{a}$ secteurs, chasses automatiques et horloge hydraulique, confort du gardien, commodité d'accès.
\end{abstract}

L'utilisation plus poussée des sites, en multipliant les prises secondaires, confère à ces derniers ouvrages, quoique d'importance parfois modeste, un rôle de premier plan dans la bonne marche d'une installation.

Souvent, et ce sera le cas à Roselend, ces prises servent à l'alimentation d'un réservoir; la valeur élevée de l'eau captée fait alors apparaître comme d'autant plus rentable la recherche d'un fonctionnement sûr et d'un rendement élevé, que la satisfaction de ce double objectif peut n'entraîner qu'un supplément d'investissements minime. De simples améliorations de

\begin{abstract}
Situation, role, brief description and special operational fealures of the various water intakes constructed as part of the Arvan and Aussois schemes (Savoly Alps). Discussion of those points whose importance has been shown up or confirmed since operation began, such as previous knowledge of the solid and liquid discharges, adaptability to the site and development of the bed upstream, characteristics of the screens, limitation of the discharges from the water intakes, recessity of washing out the sediment from time to time, position of the desander, possibility of using the transport tunnels as additional desilting canals, efficiency of flushing systems and necessily of having sector gates, automatic flushing systems and hydranlic clock, comfort of the keeper, accessibilit!l.
\end{abstract}

détail apportées à ces ouvrages et une meilleure définition des consignes d'exploitation permettent, dans beaucoup de cas, de résorber un déficit de production de plusieurs millions de $\mathrm{kWh}$.

Prenant nos exemples dans les aménagements récents des chutes de l'Arvan et d'Aussois, nous nous sommes attachés, dans les pages qui suivent, à illustrer ce fait avec le désir de préciser, en outre, les intéressantes perspectives qu'ouvre, sur le plan énergétique, un domaine d'études sur lequel les auteurs se sont montrés, à notre connaissance, particulièrement discrets. 


\section{GÉNÉRALITÉS}

\section{SITUATION ET ROLES DES DIFFERENTES PRISES ÉTUDIEES DANS LE CADRE DES AMENAGEMENTS DE L'ARVAN ET D'AUSSOIS}

Seconde, par l'âge, de la région Alpes II (19461949), la chute de l'Arvan, de $580 \mathrm{~m}$ de hauteur et d'un débit équipé de $6 \mathrm{~m}^{3} / \mathrm{s}$, comprend, outre la prise principale de l'Arvan (b.v. $100 \mathrm{~km}^{2}$ ), deux prises secondaires du type en-dessous sur les torrents du Pradin $\left(17 \mathrm{~km}^{2}\right)$ et du Gilbert $\left(14 \mathrm{~km}^{2}\right)$.

Il s'agit d'un aménagement au fil de l'eau, donc peu équipé $\left(40 \mathrm{l} / \mathrm{s} / \mathrm{km}^{2}\right)$ où les prises secondaires n'ont, en été, qu'un rôle complémentaire vis-à-vis de la prise principale.

Ces prises échappent donc, de ce fait, à la plupart des sujétions liées à l'économie d'eau en période de charriage et leur intérêt réside principalement dans leur bonne adaptation à des sites difficiles, quoique d'altitude moyenne (1 200).

L'aménagement d'Aussois (première mise en service novembre 1950) draine, à la cote 2000 , les eaux du Doron de Termignon et de ses principaux affluents rive droite: Letta, Arpont et Pingon, Grand Pyx, à l'aide d'une galerie à écoulement libre de $12 \mathrm{~km}$ de longueur, équipée de 12 à $15 \mathrm{~m}^{3} / \mathrm{s}$.

Ces eaux, actuellement dérivées dans la retenue de Plan d'Aval (4 millions de $\mathrm{m}^{3}$ ), seront ultérieurement ramenées dans les ouvrages en charge du Mont-Cenis par un siphon court-circuitant Plan d'Aval.
Ce fait imposera, en deuxième étape, une décantation plus poussée que celle que nous avons réalisée actuellement et le complément de décantation nécessaire sera fourni, ainsi que nous l'envisageons par ailleurs à Roselend, par un dessableur général installé en bout de galerie.

Au point de vue hydrologique, le bassin versant du Doron de Termignon à la prise principale d'Entre-deux-Eaux atteint $100 \mathrm{~km}^{2}$, mais avec un assez faible pourcentage glaciaire (de l'ordre de $15 \%$ ). Au contraire, celui de chacun des divers affluents du Doron ne dépasse pas $10 \mathrm{~km}^{2}$ environ mais le pourcentage de glacier est, en général, très élevé (50\% en moyenne). En période de fonte glaciaire les apports baisseront à Entre-deux-Eaux et la galerie, devenue surabondante, pourra capter la presque totalité des débits affluents, pourtant à leur maximum.

On sera donc amené à perdre le minimum d'eau d'autant plus qu'en fin d'été celle-ci a une valeur énergétique accrue.

Ces deux nécessités, d'ailleurs contradictoires, de capter presque tous les apports et de dériver le minimum de matériaux solides, jointes aux grandes difficultés d'accès, nous ont guidé dans l'établissement des projets et notamment dans le choix de décanteurs à fonctionnement discontinu.

\section{PREMIERE PARTIE}

\section{DESCRIPTION SOMMAIRE ET PARTICULARITÉS DE FONCTIONNEMENT}

\section{$1^{\circ}$ Prise d'eau du Pradin (Arvan).}

Ce torrent, qui draine un bassin liasique partiellement recouvert de moraines, a des eaux à peu près constamment chargées en matériaux solides et est, en outre, sujet à des débâcles de laves qui sont si souvent la règle en Maurienne. Ces circonstances nous ont conduits :

$1^{\circ}$ A diriger ce torrent dans le bassin de décantation de la prise par un tunnel spécial au lieu de le ramener directement dans la galerie d'amence, cependant beaucoup plus proche.

$2^{\circ}$ A prévoir une prise du type en dessous particulièrement robuste, capable de supporter sans dommage le passage de blocs de plusieurs lonnes.

Dans ce but la portée de la grille, dans le sens des barreaux, a été réduite au strict minimum compatible avec le débit à absorber (environ $200 \mathrm{l} / \mathrm{s}$ par metre de seuil) et cette grille 


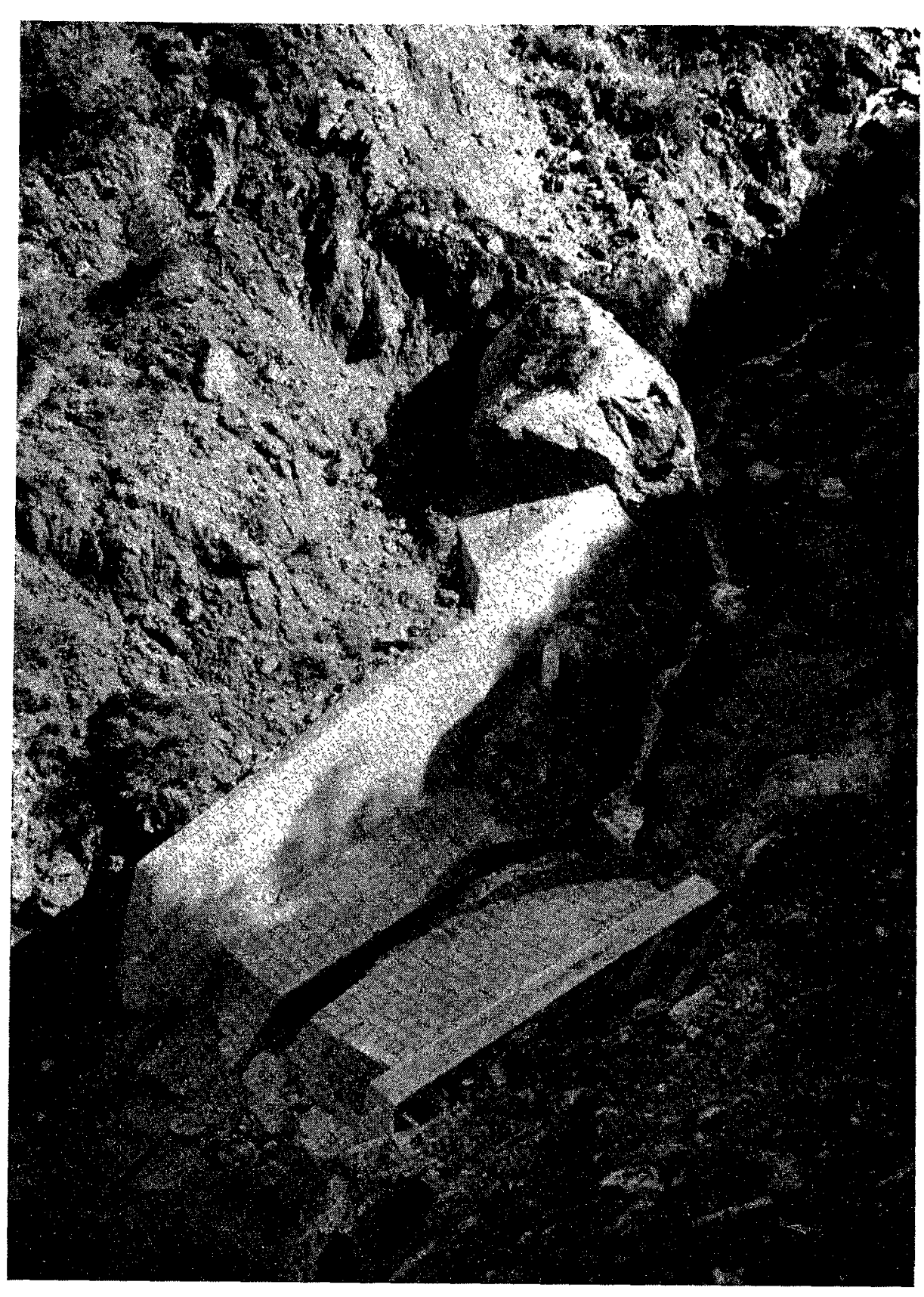

Рното 1 PRISE DU GILIBETT

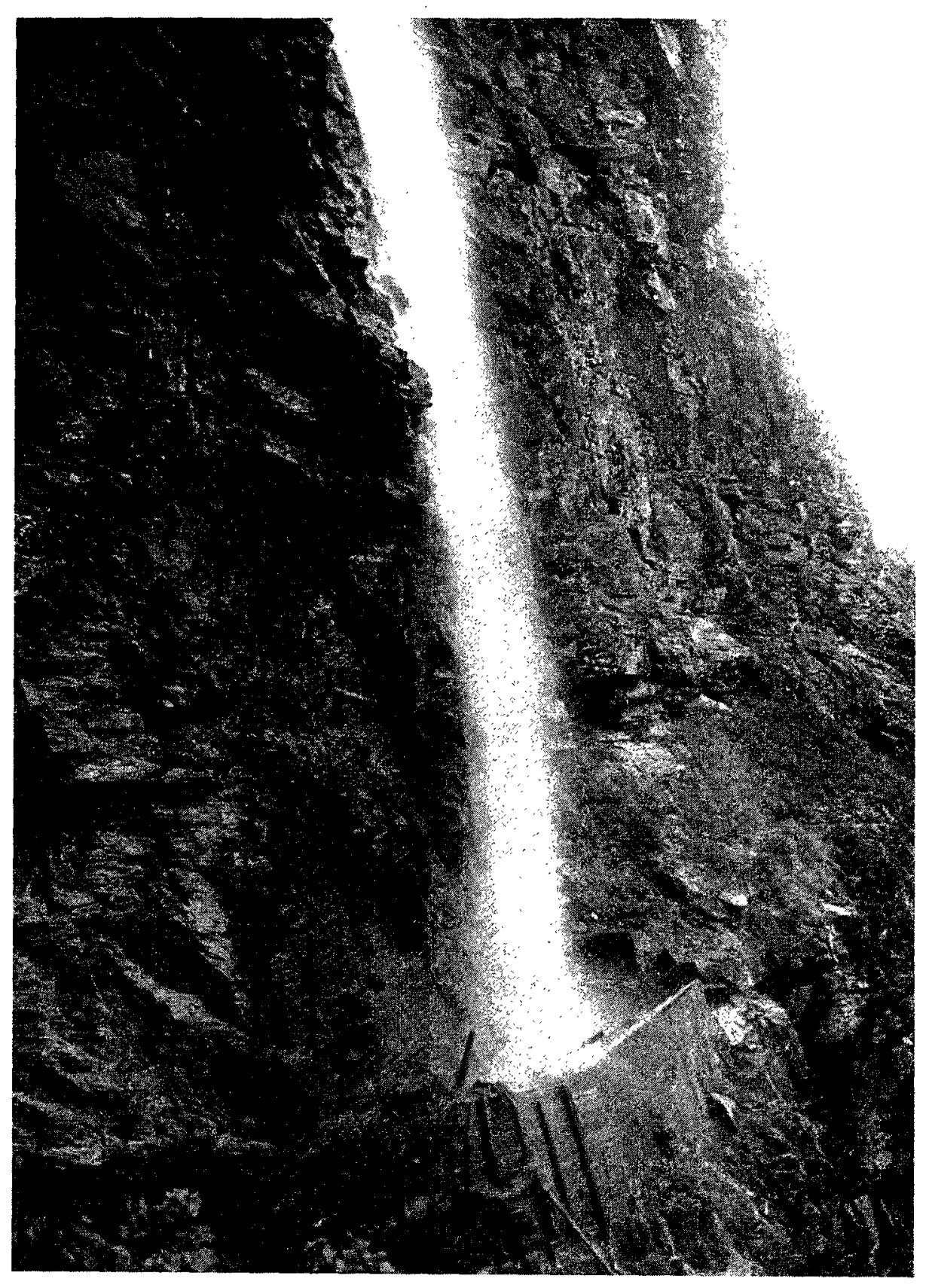

Рното 2

Prise auxiliaire du Miribet 


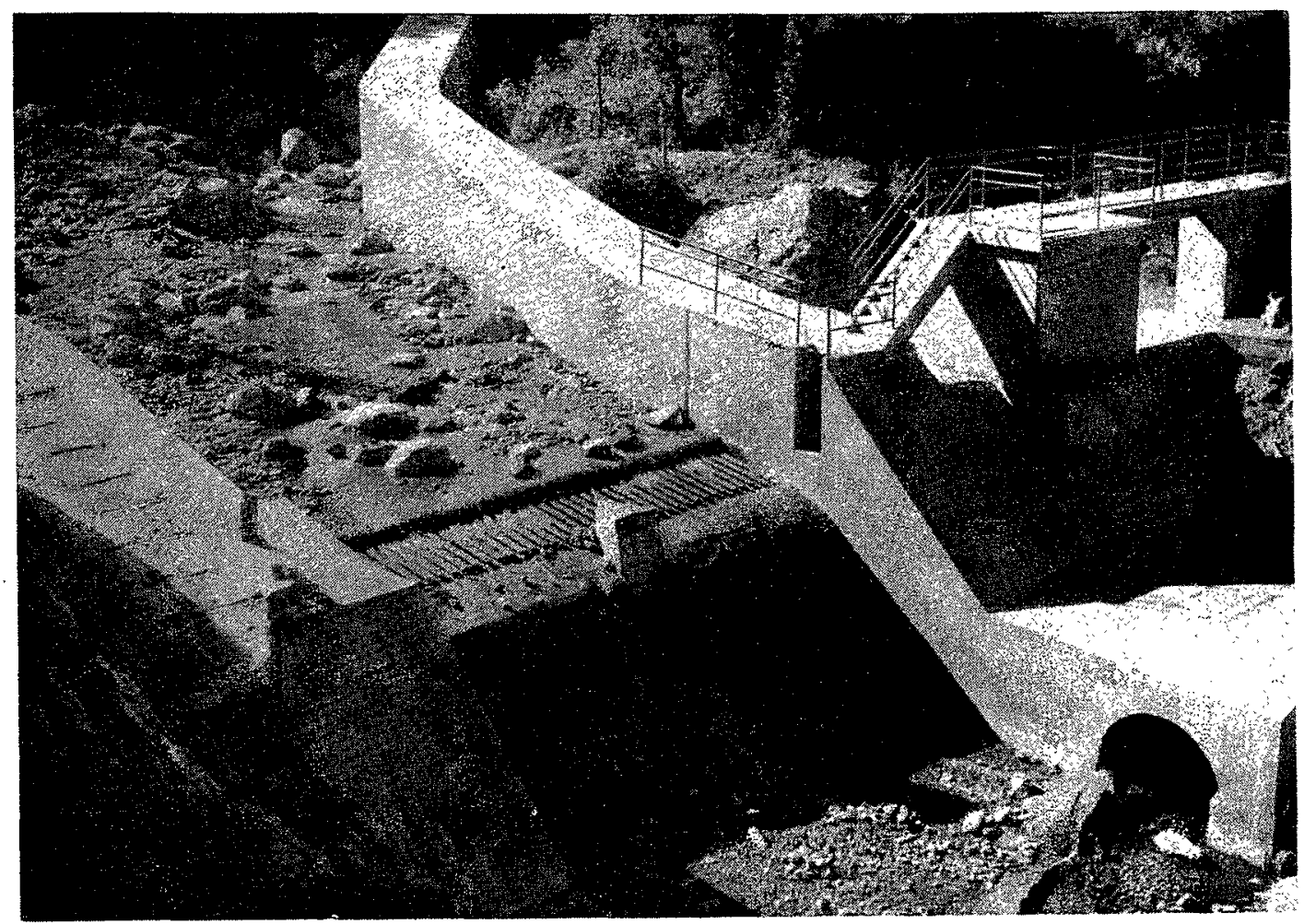

Рното 3

Puise d'eaU du Pradin

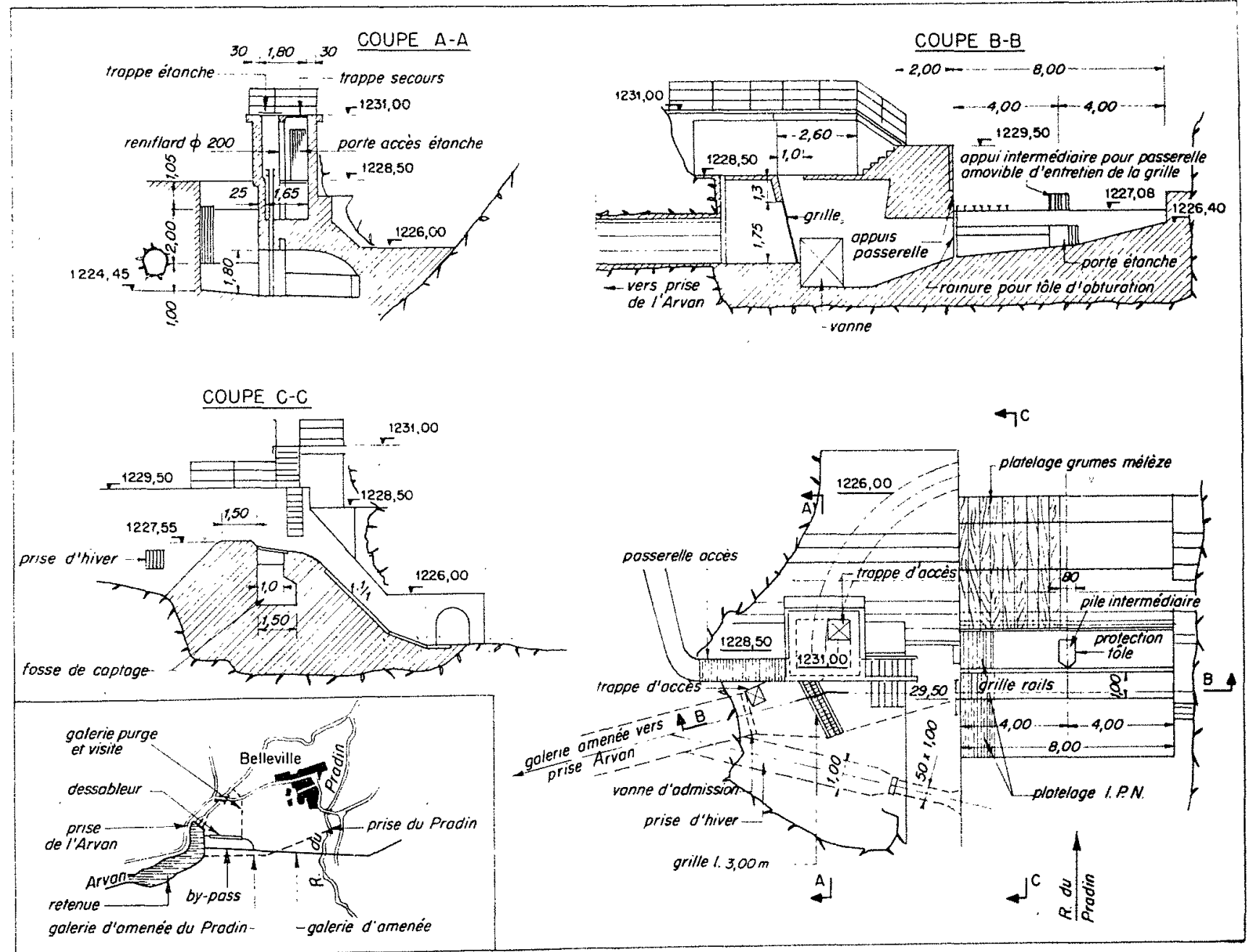

Planche 1. - Chute de l'Arvan. - Prise d'eau du Pradin 
a été au contraire allongée transversalement au torrent, autant que la topographie le permettait. Deux bajoyers en béton massif et fondés au rocher, lequel, circonstance heureuse, existe à l'emplacement des ouvrages mais pas en-deçà, délimitent le chenal offert aux crues. Par ailleurs, un platelage, métallique à la partie supérieure du barrage, et formé de mélèzes le long du coursier, protège efficacement le béton contre une érosion trop rapide.

Ainsi que nous l'avons souligné précédemment, cette prise est peu utilisée en été et encore n'est-il nécessaire, dans ce cas, de ne dériver qu'une fraction du débit : les ouvrages de dégravage sont donc très sommaires et leur rôle consiste surtout à sélectionner les débits en rejetant directement dans le Pradin les excédents d'eau et de matériaux solides dont il a paru préférable de ne pas saturer la retenue de l'Arvan.

Une prise d'hiver, - en fait, difficilement utilisable en raison des matériaux qui garnissent

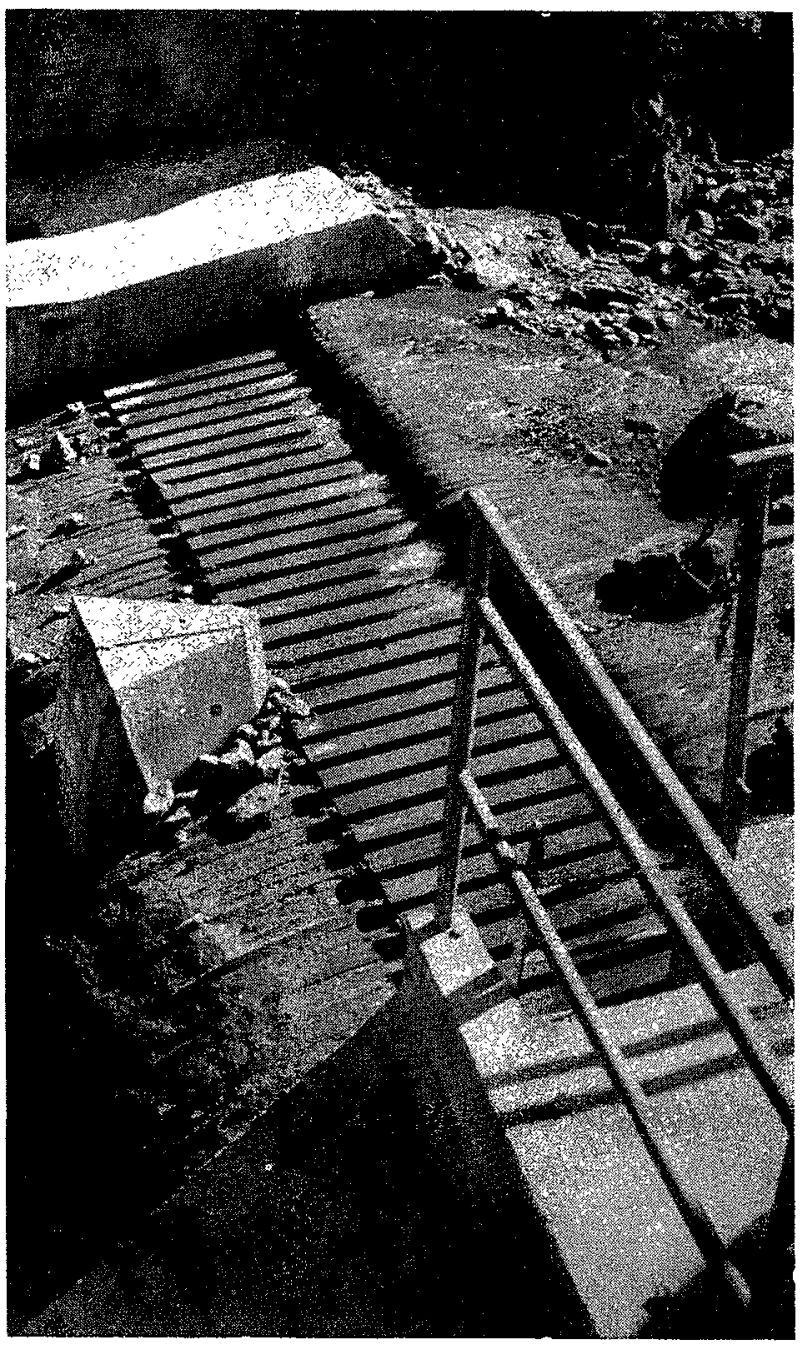

Photo 4. - Prise du Pradin

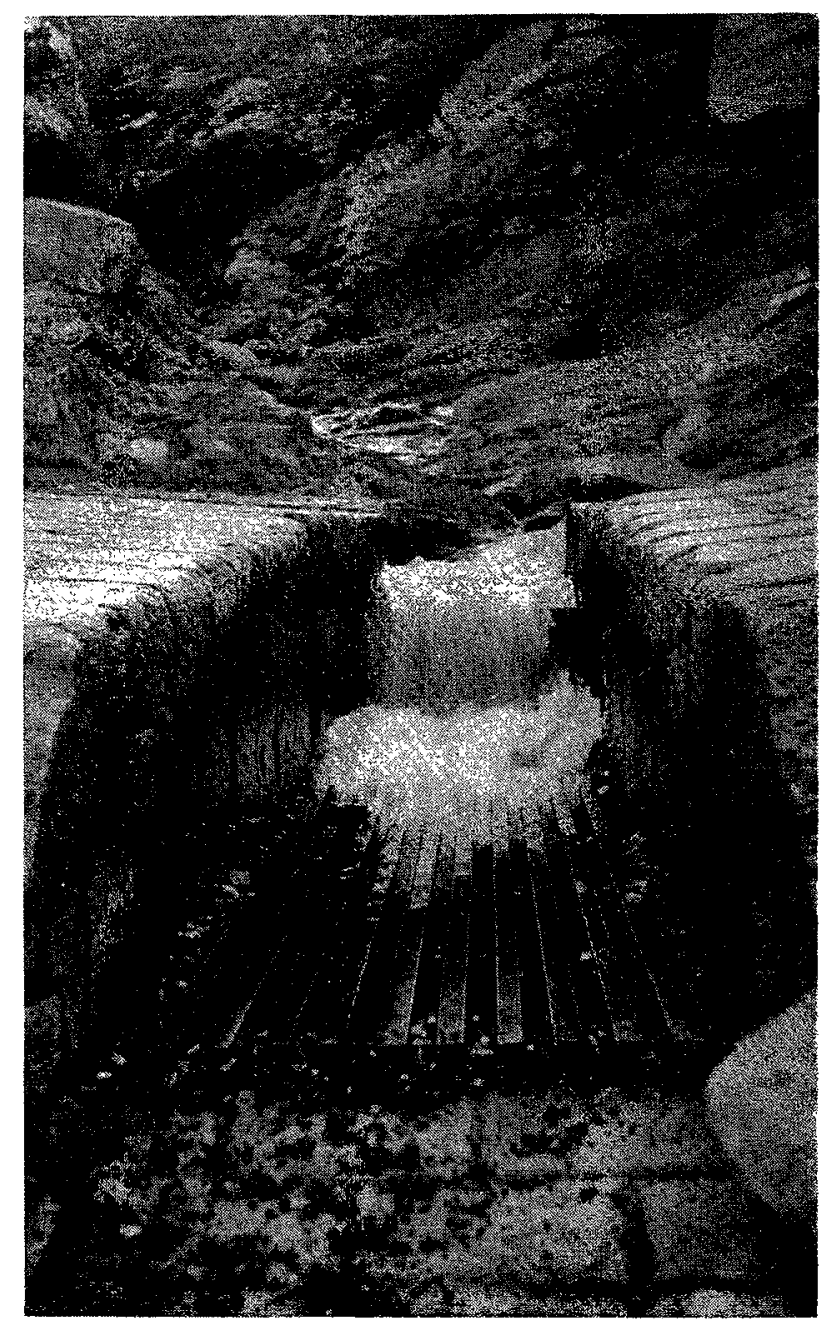

Photo 5. -..- Prise du Gilbert

le lit - a pour rôle second de by-passer les ouvrages de prise lors des nettoyages ou réparations de ceux-ci.

Les remarques suivantes, d'importance diverse, peuvent être faites après quelques années d'exploitation :

- Longueur insuffisante des rails, $1,50 \mathrm{~m}$ ent été préférable à $1 \mathrm{~m}$;

- Profil insuffisamment arrondi, les ailes des patins ayant été simplement rabattues à la forge;

- Fosse à cailloux trop petite, eu égard à la présence, immédiatement à l'aval, d'une grille fine limitant le calibre des matériaux pouvant pénétrer dans le tunnel. 


\section{$2^{\circ}$ Prise d'eau du Gilbert (Arvan).}

Ici la prise en dessous s'imposait à l'évidence, et bien que la confirmation d'un tel choix eût été superflue, celle-ci nous fut apportée en cours de travaux par une débâcle de laves d'une intensité remarquable qui rasa tous les ouvrages provisoires. Le flot de boue qui remplissait la gorge sur $20 \mathrm{~m}$ de hauteur transportait, au dire des témoins, des blocs de pluš́eurs dizaines de tonnes qui fjottaient en surface.

L'intensité de ce phénomène nous incita, non seulement à établir la prise elle-même sans aucune superstructure, mais à proscrire également tous ouvrages extérieurs, fût-ce dans un rayon relativement éloigné de la prise; il nous conduisit, par ailleurs, à prévoir l'accès aux ouvrages par un puits spécial débouchant audessus de la gorge.

A l'image du Pradin, mais dans le sens per- pendiculaire, la grille a été étirée en longueur de façon à réduire au maximum sa largeur. En outre, cette grille a été placée au fond d'une rainure de captage, étroite et profonde, qui la protège, lors des crues, contre le contact des plus gros blocs. Cette rainure a été établie sensiblement dans l'axe du lit mineur que le torrent avait lui-même creusé dans le rocher, assurant une bonne alimentation de la prise à laquel.e contribue également la pente transversale des deux bordures latérales en pierres de taille.

Les autres organes de cette prise, implantés comme nous l'avons dit en souterrain, n'offrent rien de bien remarquable par leur conception ni par leurs dimensions, exception faite du puits de raccordement avec la galerie dont la grande hauteur est liée au choix de l'unique emplacement de prise poss.ble. En tête, ce puits est muni d'une grille fine qui, lors des crues avec forts charriages, se bouche, limitant ainsi les

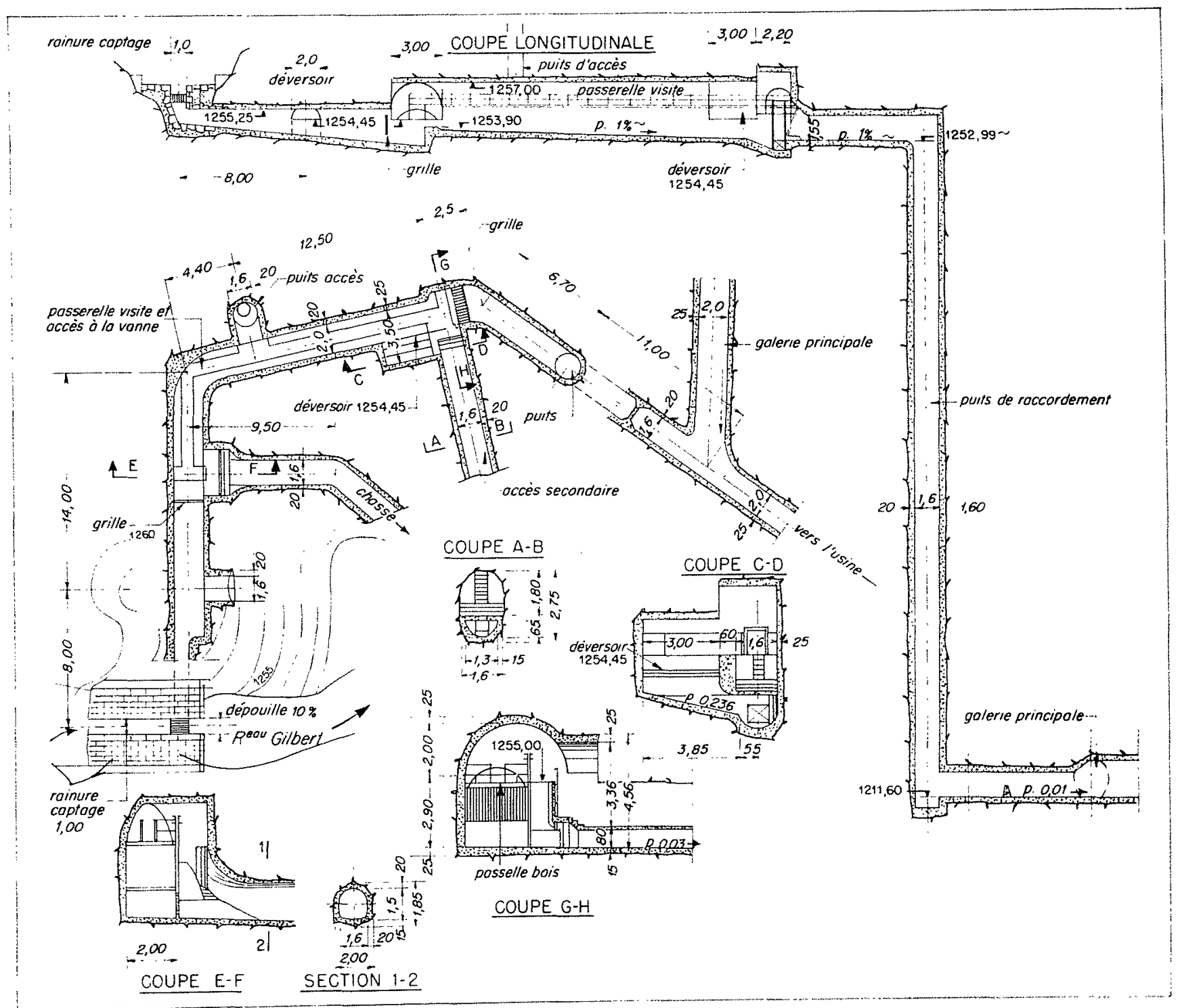

Planche II. - Chute de l'Arvan. - Prise d'eau du Gilbert 
quantités de matériaux solides entrant dans la galerie.

\section{Observations d'exploitation :}

- Coincement de certains blocs dans la rainure de captage qui aurait dû présenter une dépouille plus importante que celle qui avait élé ménagée;

- Obstruction fréquente de la grille de tranquil isation située à l'origine du dégraveur;

-- Dégraveur de dimensions insuffisantes, obligeant à des chasses répétées en ćtẻ ( 3 par jour), ou laissant passer des cailloux dans le dessab'eur;

-- Entrainement d'air relativement important dans le puits dû à une longueur de désaération insuffisante, le niveau piézométrique ne s'établissant en effet que tout à fait à la base du puits.

Dans sa conception d'origine, la chute de l'Arvan devait comporter une troisic̀me prise auxiliaire sur le torrent du Merderel; compte tenu de la description aue nous venons de faire du site $d u$ ruisseau Gilbert, nous laissons le lecteur imaginer quelles devaient être les conditions de captage de cet autre torrent quand il saura que nous avons renoncé à tenter de le prendre.

\section{$3^{\circ}$ Prise de la Letta (Aussois).}

Nous avons utilisé, pour l'implantation de cet ouvrage, une «marmite» naturelle du torrent formant fosse de captage sur laquelle la grille a été simplement posée après rég’age des appuis.

Une autre « marmite », distante de quelques mètres à l'amont, a constitué le point de départ tout indiqué d'une dérivation provisoire que l'étroitesse de la gorge et l'obligation de bétonner en été, donc en hautes eaux, avait rendue nécessaire.

Constituée de rails d'assez fort module pour résister aux chutes de blocs et aux avalanches, la grille présentait à l'origine un écartement entre barreaux de $5 \mathrm{~cm}$ pour $11 \mathrm{~cm}$ de plein (largeur du natin de rail), intervalle jugé suffisant pour éviter l'engravement en été et le gel en hiver.

Pour limiter les rejaillissements observẻs lors des premiers essais, par suite de l'impact direct de la cascade sur les patins de rails, cet écartement dut être notablement auọmenté, mais surtout, les rails furent coiffés d'un cvlindre de forme plus hydraulique, et l'écoulement \& l'amont de la grille fut un pea réğularisé par aménagement du lit.

Compte tenu de ces modifications, on peut considérer la grille actuelle comme satisfaisante.

La gril'e est suivie d'un module à masque qui évite, lors des crues, que des quantités d'eau trop importantes susceptibles de perturber le fonctionnement du dessableur, avant d'être de toute façon déversées ne pénètrent dans la prise. Dans le cas particulier de la Letta, nous avons prévu de pouvoir modifier le réglage de cet appareil suivant l'époque de l'annće, de façon à profiter au maximum, en fin d'été, des débits de fonte glaciaire.

Une vanne de chasse fermant la partie inférieure du module à masque peut maintenir l'avant canal propre et permet, en outre, la mise hors d'eau des ouvrages aval.

Au niveau de la galerie principale, soit $25 \mathrm{~m}$ plus bas, on trouve une galerie de décantation de $65 \mathrm{~m}$ de longueur à $3,5 \%$ de pente en radier et une chambre souterraine contenant les déversoirs de réglage et la vanne de chasse; celle-ci a des dimensions suffisantes pour meltre à écoulement contrôlé par l'amont la galerie de chasse et assurer ainsi son bon neltoyage. Un balardeau situé à l'amont de la chambre empêche les retours d'eau de la galerie principale lors des chasses.

Il est intéressant de noter que ces dispositions, et en particulier le volume de stockage dont les dimensions ont ćlć imposées par la topographie et les nécessilés de travaux, n'ont rien de surabondant : l'avant canal de la partic supérieure ne joue pas un ròlc très important mais l'expérience a montré đu'il suffisait d'un temps extrêmement court pour remplir toutes les galer:es de purge : 1 jour ou 2 , ou même $1 / 2$ journée.

Quant au puits de raccordement de $1,50 \mathrm{~m}$ de diamètre, pour lequel nous avions songé primitivement à un revêtement affectant au moins la partie inférieure, nous avons eu la satisfaction de constater qu'il n'y a eu aucun inconvénient à le laisser entièrement brut et que le rocher des parois et le fond se sont simplement po'is sous laction des cailloux. Ici la désaćration ne nous causait pas de souci et l'orifice circulaire prévu en haut du puits avait surtout pour objet de protéger les parois contre l'im. pact direct de l'eau.

\section{$4^{\circ}$ Prise du Grand Pyx (Aussois).}

Le Grand Pyx coule à la cote de la galerie dans une gorge d'accès difficile dont la pente, en moyenne élevée, s'atténue brusquement vers lamont, fixant ainsi une limite à la prise. 
Ces données et l'existence de deux galeries ont conditionné l'allure en plan des ouvrages : la fenêtre A-B avait été utilisée pour le percement de la galerie principale, et la galerie C-D avait été forée au début des travaux.

La galer:e de prise comprend, à l'intersection de la galerie C-D, un piège à cailloux, à purge continue, constituée par une saignée recouverte d'un masque, terminée par une vanne de $\varnothing \mathbf{4 0 0}$ (vanne de série). En fait, d'ailleurs, ce dispositif très simple est rarement utilisé : la prise est à l'altitude 2000 et on ne peut pas envoyer quelqu'un, plusieurs fois par jour, pour l'ouvrir quand il $y$ a des excédents, et le fermer quand le débit de la galerie ou du torrent diminue : il faudrait, en outre, le savoir très vite, ce qui n'est

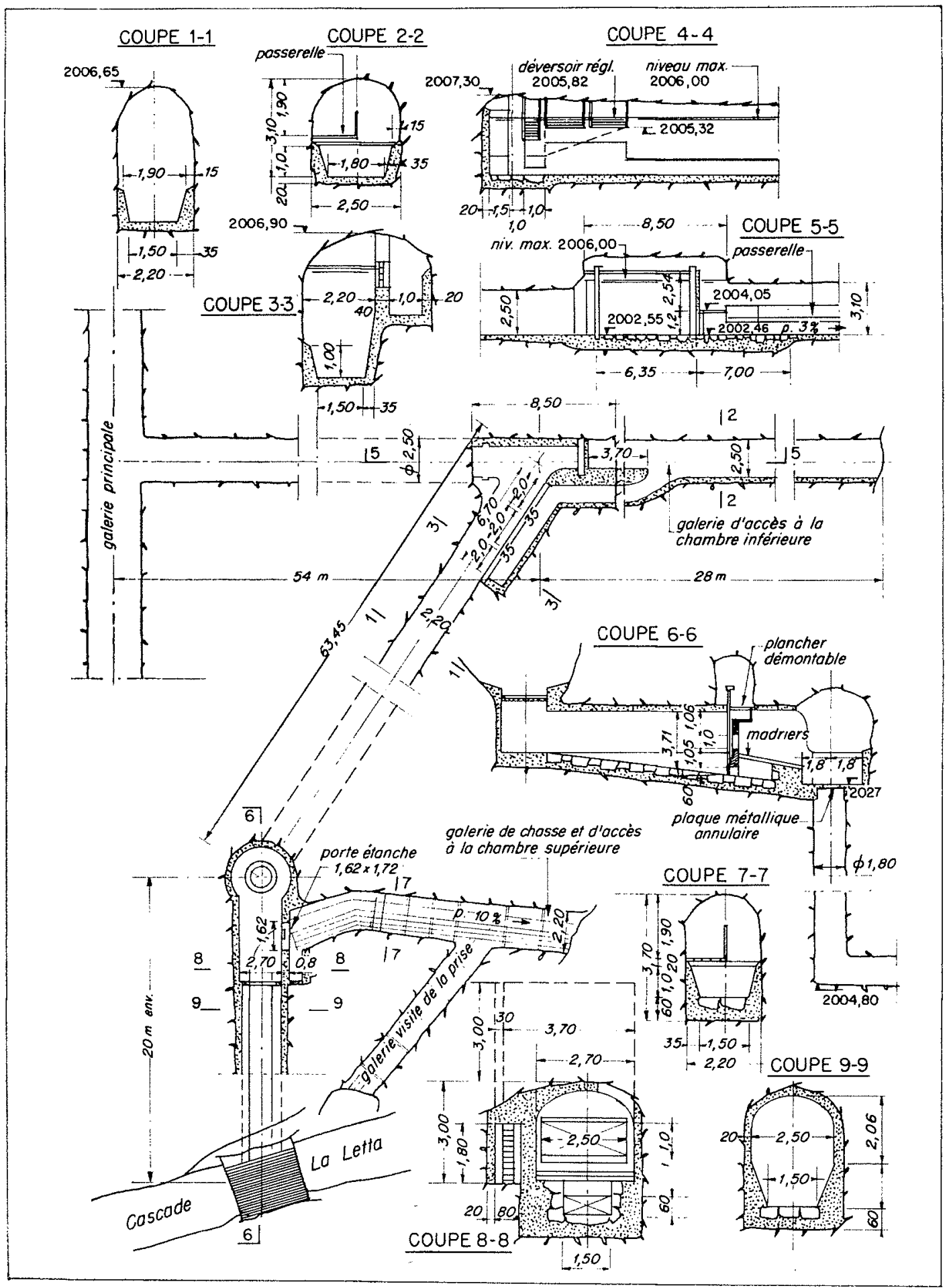

Planche III. -- Chute d'Aussois. - Prise d'eau de la Lelta 


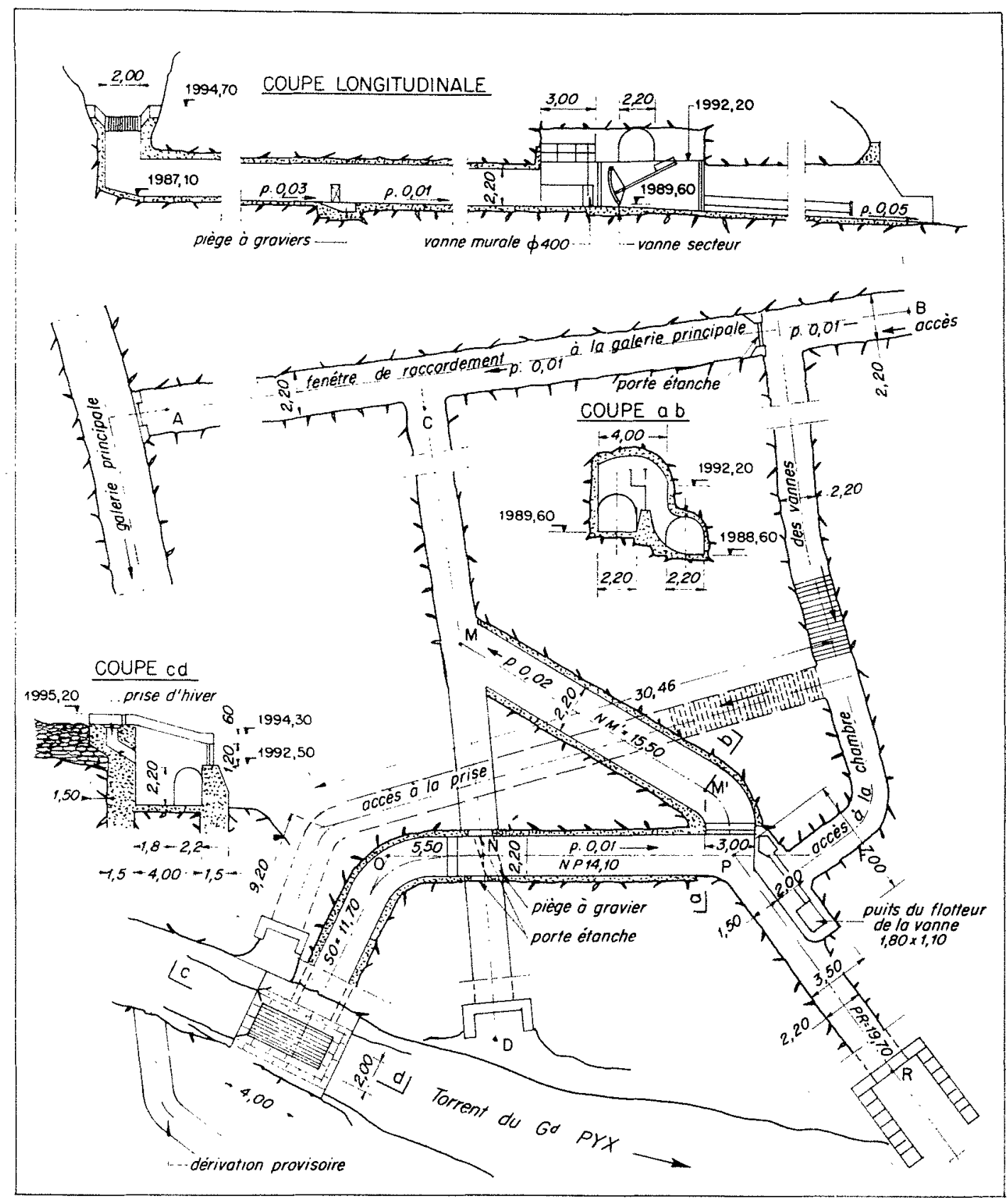

Plaxche IV. - Chute d'Aussois. - Prise d'eau du Grans Pyx

pas des plus facile. Néanmoins, son fonctionnement semble correct.

La galerie (dont la pente est de 1,5\%) aboutit ensuite à la chambre des vannes. Elle est obstruée en bout par une vanne à secteur automatique, asservie au niveau amont. En marche normale, cette vanne évacue les excédents décelés par une surélévation du niveau - par le fond, donc de l'eau plus chargée que celle qui serait évacuée par un déversoir de surface. Elle n'ẻtait pas destinée à faire des chasses automatiques qui nécessitent la mise en écoulement «contrôlé par l'amont» de la galerie de purge. Bien entendu, une manouvre à main, soit par action sur le flotteur de la vanne, soit par un palan, permet de faire une chasse volontaire.

Le déversoir, qui alimente le canal d'amenée, est calé de façon qu'il puisse être noyé quand le nıvoau dans la galerie est élevé et, s'il l'est trop, la vanne se lève automatiquement pour évacuer de l'eau. Ce déversoir est traversé par un orifice pouvant ctre obturé par une « vanne d'hiver », destinée à abaisser le tirant d'eau en hiver et à augmenter la vitesse pour éviter le gel.

La longueur des canalisations raccordant ces ouvrages à la galerie principale a nécessité d'autres précautions: le tirant d'eau, en hautes 
eaux, est contrôlé par le niveau dans cette galerie, ce qui diminue beaucoup la vitesse dans les galeries de raccordement prévues à la section minimum. La vitesse étant faible, on peut craindre une décantation parasite des matériaux transportés. Afin de prévenir ce phénomène, la porte étanche obturant la fenêtre à la fin des travaux a été munie d'une vanne plate, à commande manuel e de $1 \mathrm{~m}$ sur $0,80 \mathrm{~m}$ permettant d'abaisser les lirants d'eau dans ces galeries et, par suite, de réaliser une chasse. Au raccordement galerie principale-fenêtre, un masque en bois limite les retours d'eau de la galerie pour ne pas engorger la vanne. En outre, la pente de la fenêtre, en aval de la vanne, a été augmentée pour permettre une évacuation plus commode des sables, des graviers ou même des ca:lloux déposés.

En fait, d'ailleurs, ces précautions ne se sont pas avérées inuti'es : l'ampleur du charriage du Grand Pyx est telle que, lors d'une défailance des vannes, les galeries, jusqu'au canal princi- pal, se remplissent complètement de cailloux en un ou deux jours. On risque alors une obstruction tota'e, conséquence du calage trop bas de la prise imposé par la topographie, et l'ouvrage reste d'une exploitation difficile.

\section{$5^{\circ}$ Prise du Thibaut (Aussois).}

Le bassin versant atteint $6 \mathrm{~km}^{2}$. Le type de prise est analogue à celui de la Letta; l'emplacement favorable du captage ćtant trop haut par rapport à la galerie, les ouvrages sont divisés en deux parties: Ia partie supćricurc comprend la grile prolongée par un déversoir qui permet à la hauteur d'eau sur les grilles de ne pas trop augmenter en cas de crue, et par suite á la prise de ne las trop absorber les crues. II a ćté nécessaire d'amćnager sérieusement le lit du torrent à l'amont, car il ressembla ‘t plus à une suite de cascades qu'à un lit bien délimité. Il est possible de batarder l'amont des grilles pour dériver l'eau sur le déversoir déjà signalé.

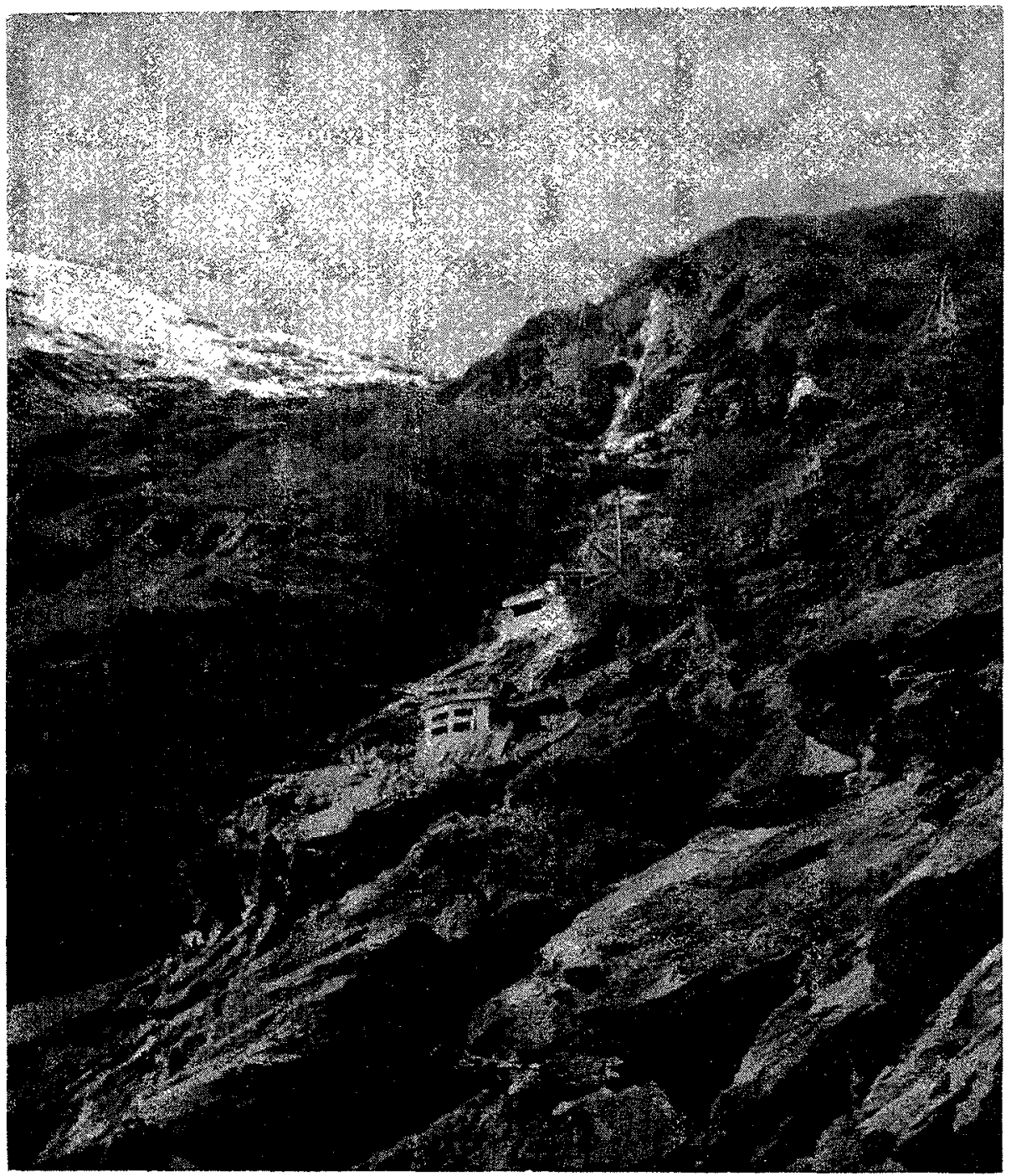




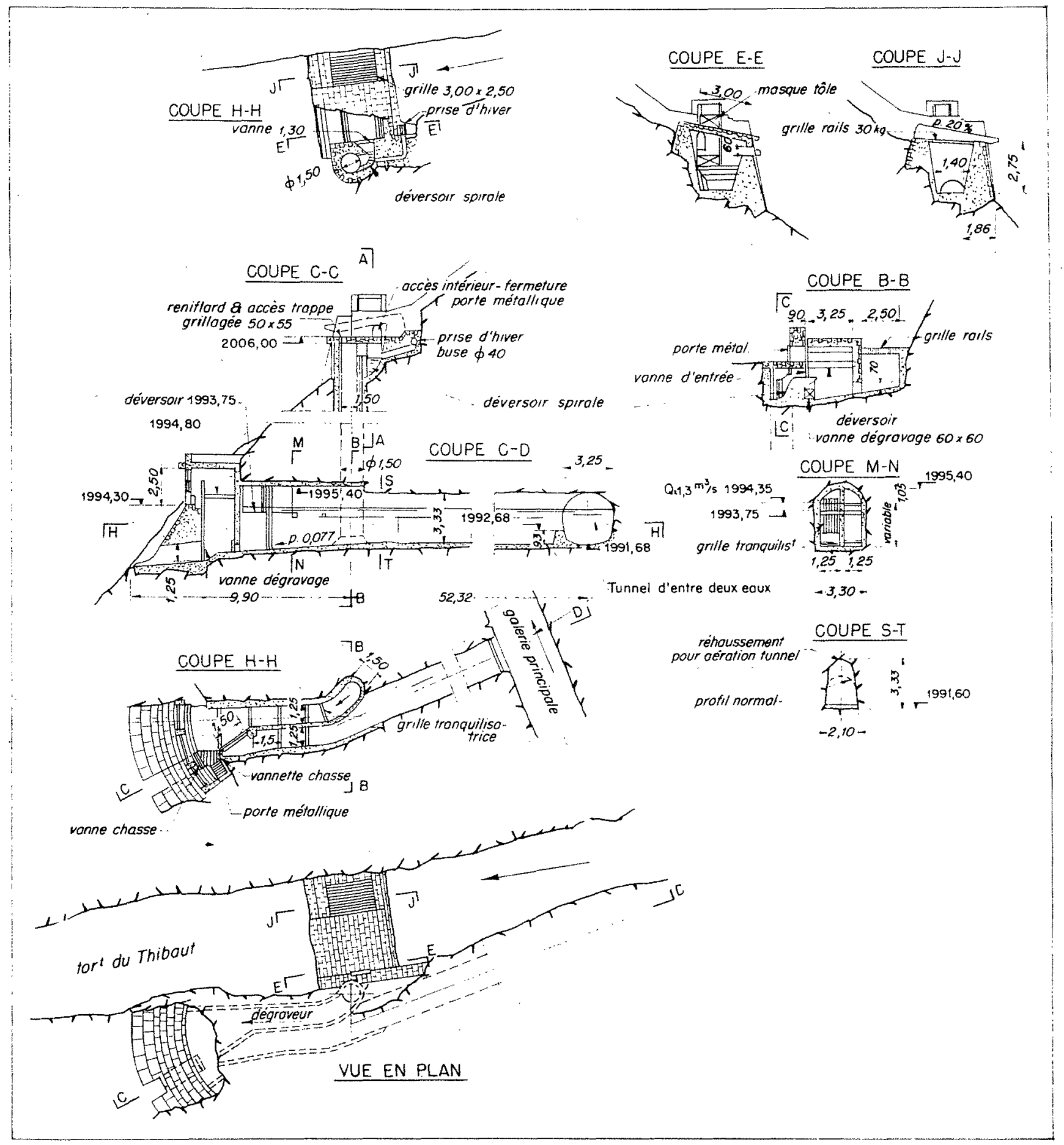

Planche V. - Chute d'Aussois. - Prise d'eau du Thibaut

Les grilles sont suivies d'une chambre en béton et pierres de taille, qui comprend :

- Une vanne sur le canal de dérivation aval quí joue le rôle d'un module à masque et est, par suite, jumelée avec un déversoir;

- Une vanne de chasse précédée d'un canal à forte pente qui permet de purger les matériaux solides.
La chambre débite dans un puits revêtu d'une dizaine de mètres de haut. Afin d'examiner quelle pourrait être l'influence favorable de ce système pour le dégazage de l'eau, après entrâ̂nement de l'air dû aux grandes vitesses de chute, on a recherché dans ce puits un effet centrifuge : l'eau est amenée tangentiellement à la partie supérieure du puits, avec une assez forte vitesse, elle reste donc tangente à la paroi 
en gardant une partie de sa vitesse tangentielle. II est à noter d'ailleurs que ce système n'était pas indispensable dans notre cas, puisqu'il s'agit d'une galerie à écoulement libre où le dégazage se produit naturellement, par «décanlation ». Néanmoins, il était ainsi possible de réaliser une expérience en vraie grandeur, présentant un grand intérêt pour la chute de Roselend où le problème se posera fréquemment.

A la suite de ce puits, l'eau tombe dans un bassin de décantation placé à la partie inférieure, sensiblement au niveau de la galeriè, où le tirant d'eau est contrôlé par un déversoir placé en aval. Ce chenal est terminé par une vanne permettant d'effectuer des purges discontinues. Le dégraveur est disposé d'une façon un peu particulière par rapport à la fenêtre qui dérive les eaux dans la galerie (cf. figure). Sur ce rameau de tunnel, on a également prévu une petite vanne permettant de purger les dépôts qui se formeraient par suite des vitesses faibles correspondant aux forts tirants d'eau imposés par le niveau dans la galerie principale.

\section{$6^{\circ}$ Prise de l'Ile (Aussois).}

Le ruisseau de l'Ile n'est, en fait, qu'une des branches secondaires du ruisseau de l'Arpont, Iui-même constitué, à la cote du captage par le Thibaut.

Les autres subdivisions de ce ruisseau, au nombre de 4 ou 5 , ne sont que de simples ruisselets dont ies ouvrages de prise, encore plus sommaires que ceux de l'lle, ne seront pas décrits.

La prise de l'Ile se compose d'une simple fosse de captage avec un dispositif de chasse latérale pour le nettoyage périodique de cette fosse. Une grille fine protège contre les apports solides la conduite métallique de liaison avec la galerie principale.

On remarquera que cette conduite métallique a élé poussée jusqu'au droit de la galerie principale - alors qu'ou aurait pu songer à utiliser la tenêtre en reportant le bouchon près de l'extérieur - dans le but d'éviter toute perte d'eau dans la fenêtre et toute décantation des sables.

\section{$7^{\circ}$ Prise du Pingon (Aussois).}

La prise d'eau sur le Pingon a été exécutée au cours de l'été 1953 alors que les autres prises d'eau étaient déjà en exploitation depuis un an ou deux.

A l'origine, il avait été prévu que les eaux de ce ruisseau seraient dérivées par une simple tranchée creusée à même le terrain, vers le torrent du Thibaut, de facon à capter par une seule prise les eaux des deux torrents réunis. Ce canal a fonctionné pendant deux années au cours desquelles il s'est avéré que les apports étaient beaucoup plus importants que ceux que

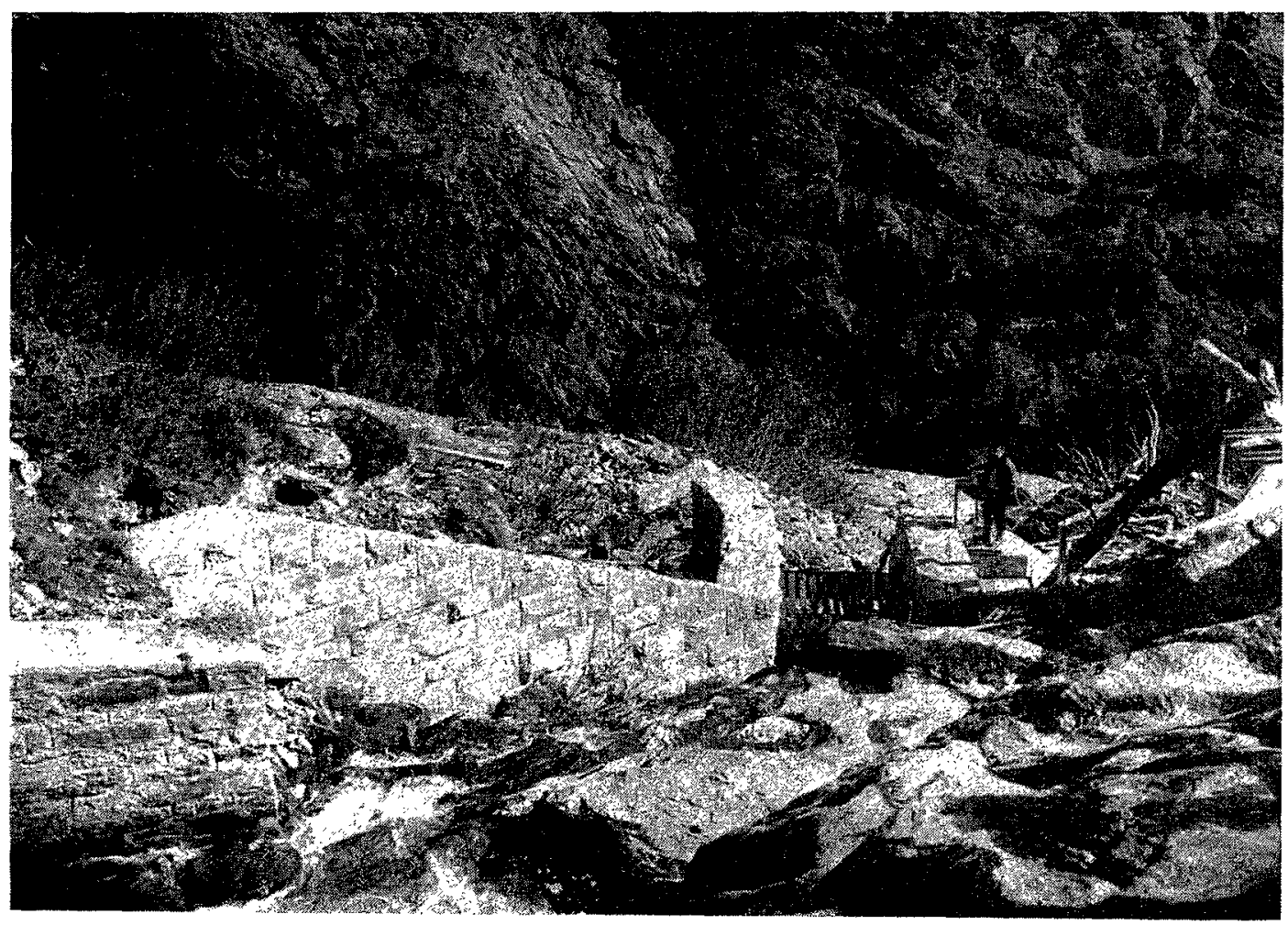




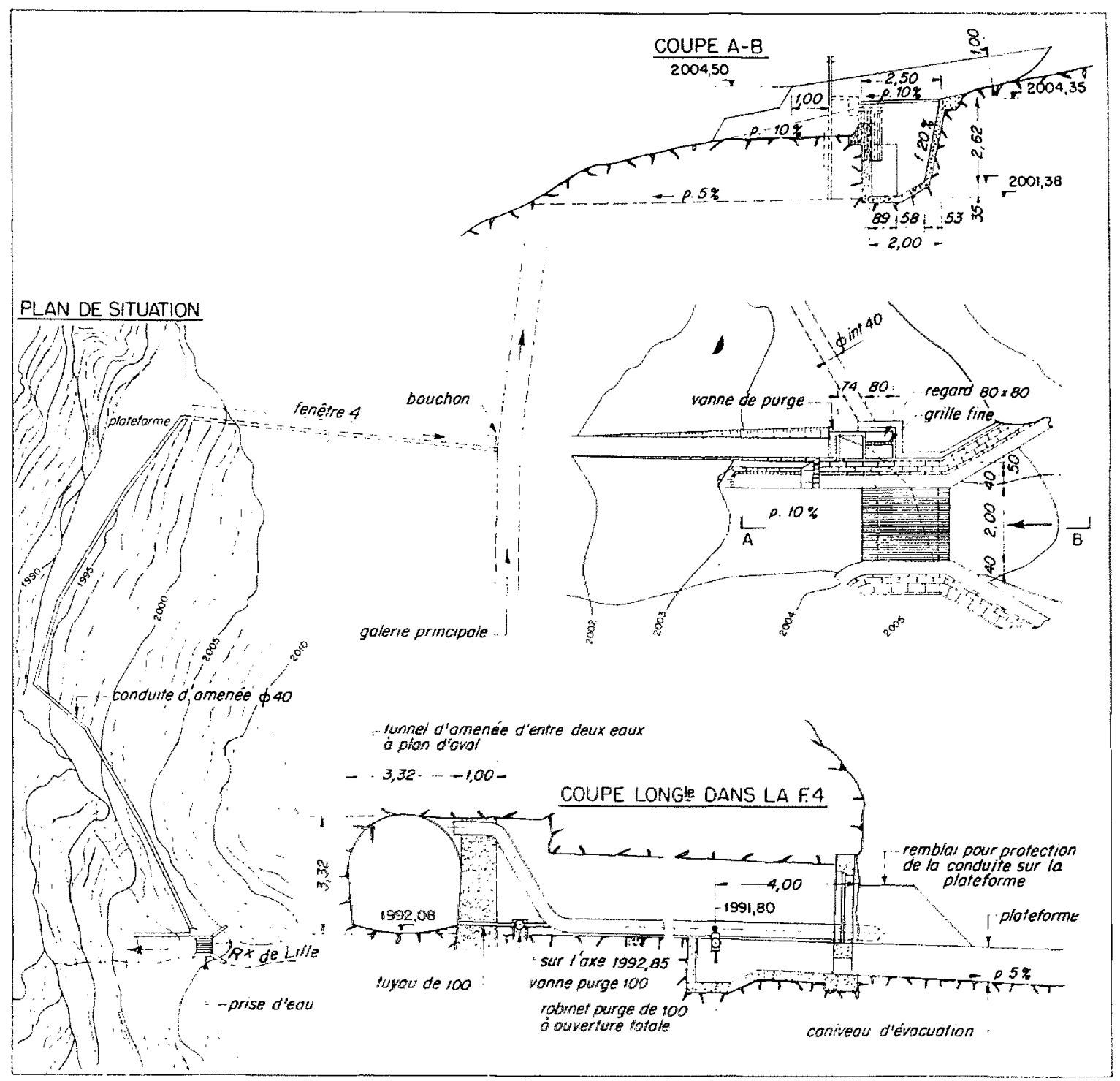

Plancae VI. -.. Chute d"Aussois. - Prise d'eau de l'Ile

l'on avait estimés ef que la prise du Thibaut était sous-équipée au moment où la quantité d'eau, amenée par la galerie principale, était insuffisante. Par ailleurs, le Pingon étant sujet à des crues assez importantes, le canal de dérivation avait nécessité des travaux de réfection et de consolidation assez onéreux. Il a done paru nécessaire de construire une prise d'eau nouvelle destinée à collecter séparément les eaux du Pingon.

L'étude de cette nouvelle prise se faisant après deux ans d'exploitation de la chute d'Aussois a pu bénéficier de l'expérience acquise. A ce moment, d'ailleurs, commençait l'étude des prises d'eau de l'aménagement de Roselend comportant une trentaine de prises situées également dans des endroits d'accès difficiles pour lesquels on étudiait un dispositif de chasse auto- matique dit par «horloge hydraulique ». Il était donc normal d'utiliser le Pingon comme banc d'essaí de ce dispositif. Voulant également éviter les ennuis rencontrés par l'utilisation des rails dans la construction des grilles de prises en dessous, des essais sur modèle réduit furent effectués dans le but de rechercher le profilé optimum ainsi que les dispositions à prendre pour éviter le bouchage des intervalles par les transports solides du torrent. Ces essais ont montré que le profil " couteau * dont nous reparlerons plus loin donnait le meilleur rendement.

II fut décidé également d'essayer un dispositif de chasse en retour utilisant les eaux de la galerie principale, ce qui permettait de disposer avec certitude du débit optimum pour réaliser la chasse dans le temps minimum. 
Les dimensions adoptées pour la grille ont été de $2,50 \times 3,00 \mathrm{~m}$. Elle est précédée d'un canal à radier lisse de même largeur et même pente et de $3 \mathrm{~m}$ de longueur. A la suite, se trouve le dégraveur proprement dit constitué par une galerie d'une trentaine de mètres de longueur, présentant un léger coude permettant aux ouvrages de s'encastrer plus profondément dans le sol. Cette galerie a une pente de $2 \%$; elle est revêtue en radier et en piédroits sur une hauteur d'un mètre en profil trapézoïdal. Elle est fermée à l'aval par une vanne secteur de $l=1,50 \times h=2,50$ à commande hydraulique. Cette galerie se prolonge à l'aval de la vanne

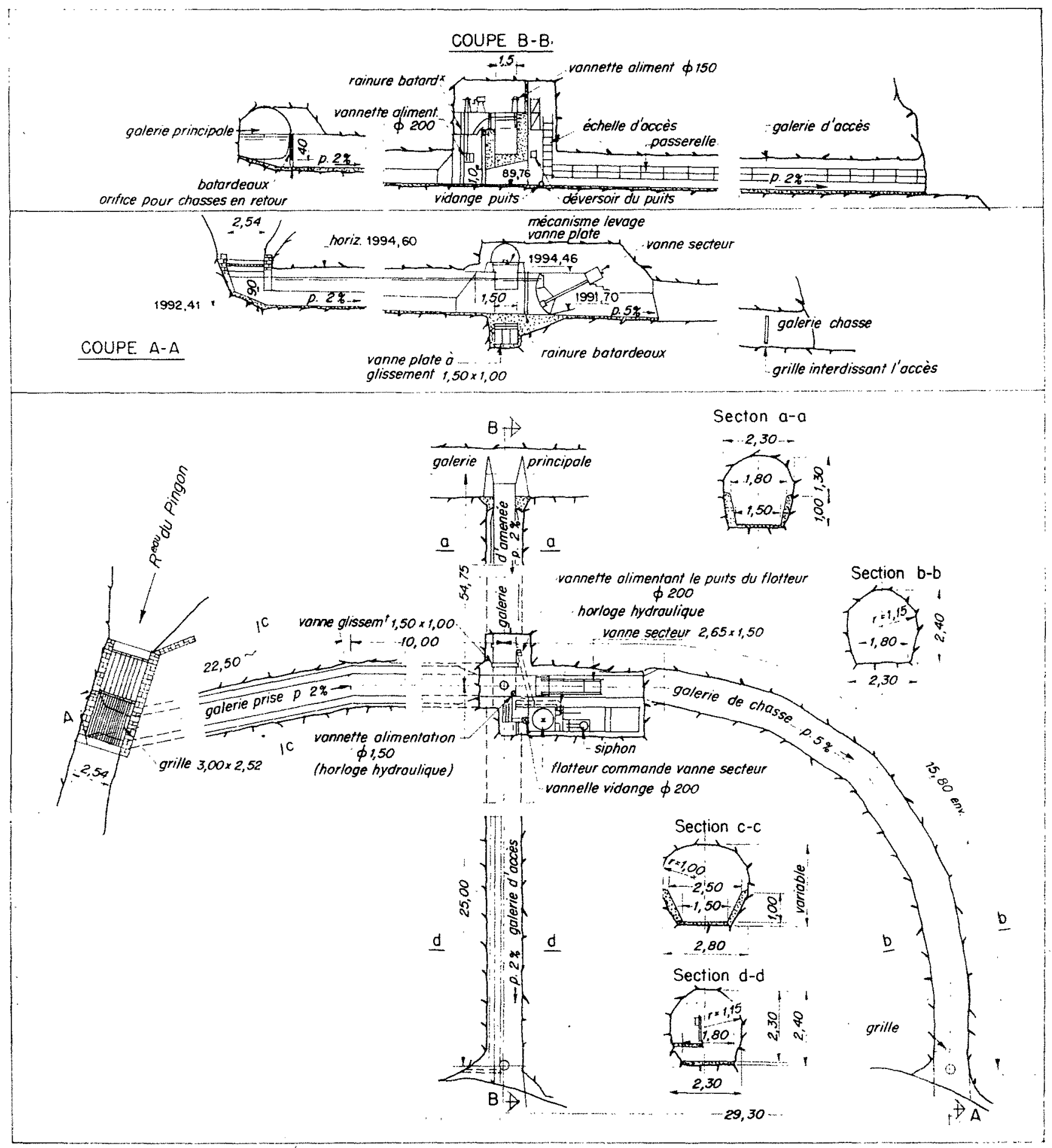


avec une pente de $5 \%$ et débouche à lair libre quelques dizaines de mètres plus loin. Toute cette partie a été laissée brute d'excavation.

Un peu à l'amont de la vanne secteur, et par en dessus, cette galerie de dégravage coupe à angle droit la fenêtre conduisant à la gajerie principale. Cette fenêtre est obturée au droit du déversoir par une vanne plate de $l=1.50 \times h$ $=1 \mathrm{~m}$ servant pour les chasses en retour. La partie située à l'aval de cette vanne, d'une longueur de $25 \mathrm{~m}$ environ, sert d'accès à la chambre des vannes au moyen d'une passerelle.

Notons que les accès à la prise ont été particulièrement soignés. Des câbles ont été posćs formant une main courante assurant la sécurité du gardien en cas de gel. Pour éviter tout courant d'air et diminuer considérablement le risque de gel en hiver, un masque placé à l'entrée du dégraveur plonge dans l'eau lorsqu'elle atteint son niveau normal, et une porte ferme la chambre de la vanne.

Mais le caractère particulier de cet ouvrage réside dans le système de chasses aulomatiques et périodiques par «horloge hydraulique» auquel nous consacrerons - - et c'est pourquoi nous n'en parlons pas ici - un paragraphe tout entier de la second partie.

\section{DEUXIEME PARTIE}

\section{PRINCIPAUX ENSEIGNEMENTS TIRÉS DE LA MISE EN SERVICE ET DES PREMIERES ANNEES D'EXPLOITATION}

L'expérience tirée de l'exploitation des prises d'eau que nous venons de décrire nous a permis de dégager un certain nombre d'idées que nous nous proposons de développer dans ce chapitre.

Nous ne parlerons donc pas du problème des prises de montagne dans son ensemble. Nous nous contenterons plutòt d'exposer un certain nombre de points bien précis sur lesquels nous nous sommes fait une opinion. Nous rappelons à ce sujet que les problèmes envisagés sont propres aux Alpes et qu'il n'est pas toujours bon de les reporter sans esprit critique à d'autres régions, même aux Pyrénées.

\section{$1^{\circ}$ Connaissances des débits solides et liqui- des. Utilité de mesures et d'enquête préalables.}

Nous pensons qu'il est bon, tout au moins dans les Alpes, d'insister sur l'intèrêt primordial qu'il y a d'avoir une aussi bonne connaissance que possible des débits liquides et solides de chacun des torrents que l'on veut capter; en effet, cette connaissance influe profondément sur la forme et le volume des prises d'eau.

La connaissance des débits liquides ne pose aucun problème si on a eu le temps, au préalable, au moins pendant une année, de placer sur le torrent une station de jaugeage. Signalons, à ce propos, que la méthode chimique est maintenant parfaitement mise au point et per- met des mesures de débit précises et aussi nombreuses que possible pour tarer la station.

En revanche, la connaissance des débits solides des torrents est nettement plus difficile; elle est souvent liée au régime du torrent. Ainsi, d'une manière générale, un torrent d'origine glaciaire ou traversant des moraines transportera beaucoup plus de matériaux solides qu'un torrent de régime uniquement nival. Cette différentiation est d'ailleurs souvent difficile à faire : à Aussois, par exemple, deux torrents d'importance comparable, situés à 200 ou 300 mètres l'un de l'autre, sont, l'un de régime glaciaire (le Pingon), l'autre de régime nival (le Thibaut). En première approximation, on peut estimer qu'en crue et avec des bassins versants semblables, le Pingon transporte de 5 à 10 fois plus de sables et graviers que le Thibaut. On devine assez la répercussion importante qu'il en résulte súr le dimensionnement des organes de prise.

Signalons, à propos des torrents dont le bassin versant est partiellement glaciaire, que par suite du recul général des glaciers, les cartes que l'on utilise sont bien souvent parfaitement erronées. Une simple visite sur place permel d'ailleurs de s'en rendre compte. Le fait que les torrents issus de glaciers transportent, en général, énormément de boue et de matériaux solides, est d'ailleurs très probablement lié au recul des glaciers; en effet, ceux-ci, en reculant, libèrent des moraines instables qui sont, en été, lavées par les eaux de fonte. Remarquons en 
passant qu'il doit en être tout à fait autrement en périodes d'ávance des glaciers.

De toute façon, l'évaluation exacte des débits solides des torrents de faible importance est pratiquement impossible. La meilleure méthode a'évaluation est encore l'exploration systématique du cours amont. Avec un peu d'habitude, la compraraison du bassin versant étudié avec d'autres bassins versants de torrents déjà captés permet d'avoir une assez bonne idée des débits solides.

\section{$2^{\circ}$ Adaptation au site. - Nécessité d'aména- ger le lit à l'amont.}

\section{a) Adap'ition aU Site. - Prise par EN DESSOUS :}

En haute montagne, il convient, bien plus qu'ailleurs, d'adapter au site, autant que possible, les ouvrages extérieurs d'une prise d'eau. Il convient aussí, ćtant donné les conditions très dures de climat, de limiter au maximum le volume des ouvrages à l'extérieur et de prévoir ces ouvrages d'une solidité et d'une rusticité à toute épreuve. Nous ne saurions donner un meilleur exemple que la prise du Gilbert qui comme nous l'avous indiqué dans la description de cette prise - a été prévue pour pouvoir supporter, avec le minimum de dommages, des crues composées d'un mélange de boues et de blocs de rocher de plusieurs tonnes.

Cette prise est du type «par en dessous » et c'est, en définitive, de ce type que nous prévoyons actuellement nos prises dès qu'elles se trouvent dans un endroit un peu sauvage.

La prise de la Letta représente également un bel exemple d'adaptation au site puisque nous nous sommes contentés de recouvrir de grilles une magnifique « marmite» naturelle creusée dans un gneiss très dur.

Dans les lignes qui vont suivre, nous ne parlerons que des prises du type «par en dessous».

\section{b) NÉcESSITÉ D'AMÉNAGER LE LIT A L'AMONT DF. LA PRISE :}

Nous avons remarqué qu'en périodes de hautes eaux il y avait énormément de pertes d'eau sur les grilles par suite de la grande vitesse de l'eau à l'amont de la prise. Dans certains cas où le torrent est à très forte pente, ces pertes peuvent devenir extrêmement importantes (voir photo du Thibault). Dans des cas semblables, il est indispensable d'aménager le lit à l'amont de manière à tranquilliser le courant avant qu'il n'arrive dans les grilles; on corrige également ainsi une dissymétrie d'alimentation. Le système le plus efficace consiste, à notre avis, à créer à l'amont des grilles une petite cascade artificielle. Cette cascade aura une lorce érosive suffisante pour entretenir une vasque toujours vide de cailoux qui servira de bassin de tranquillisation.

Si le torrent coule sur du rocher à très forte pente, il suffira de creuser celui-ci comme indiqué sur le croquis.

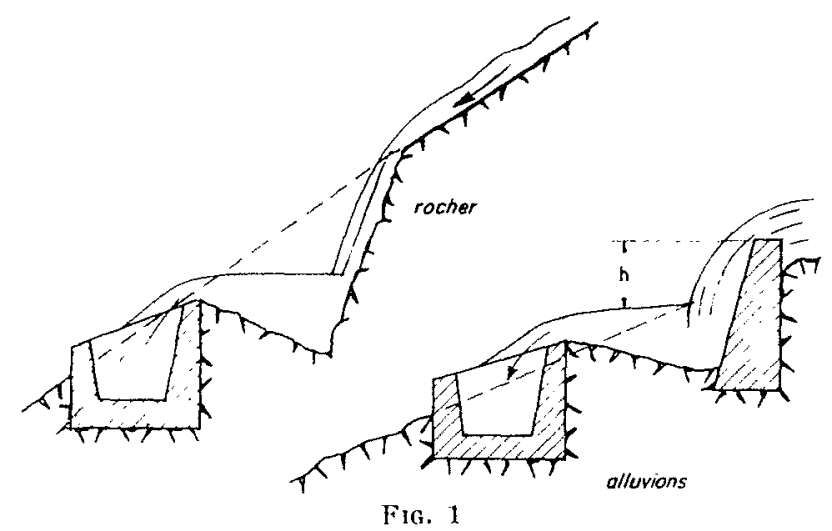

Si le torrent a un lit formé de cailloux non en place, il faudra construire, à l'amont de la prise, un mur grossier (pouvant même être formé de gabions), un peu dans le genre des travaux que font les Eaux et Forêts pour rectifier le lit des torrents soumis à une forte érosion.

Nous attirons l'attention sur le fait que la hauteur $h$ de ce mur doit être suffisante pour que la force érosive de la cascade ainsi créée puisse entrainer les cailloux les plus gros que transporte le torrent. Si cette hauteur n'est pas suffisante, il y aura dépôt de cailloux, formation à la place de la cascade d'un petit cône d'éboulis, et la cascade créée par le mur ne fera que provoquer des rejaiilissements d'eau à l'opposé du but recherché. Il est bon d'ailleurs, avant de projeter ce pré-barrage, d'explorer le lit $\mathbf{d u}$ torrent aux environs de la prise car il n'est pas rare d'y trouver des cascades naturelles suivies de vasques tranquilles qui donneront alors au projeteur une exceilente idée des dimensions à adopter.

\section{$3^{\circ}$ Forme et écartement des barreaux de grilles.}

a) Forme des barreaux de grilles :

Les caractéristiques des barreaux de grilles dépendent de la résistance mécanique que celles-ci doivent offrir, liée elle-même aux caractéristiques locales: risques d'avalanches, de débâcle, etc. Des études sur modèle réduit, réa- 


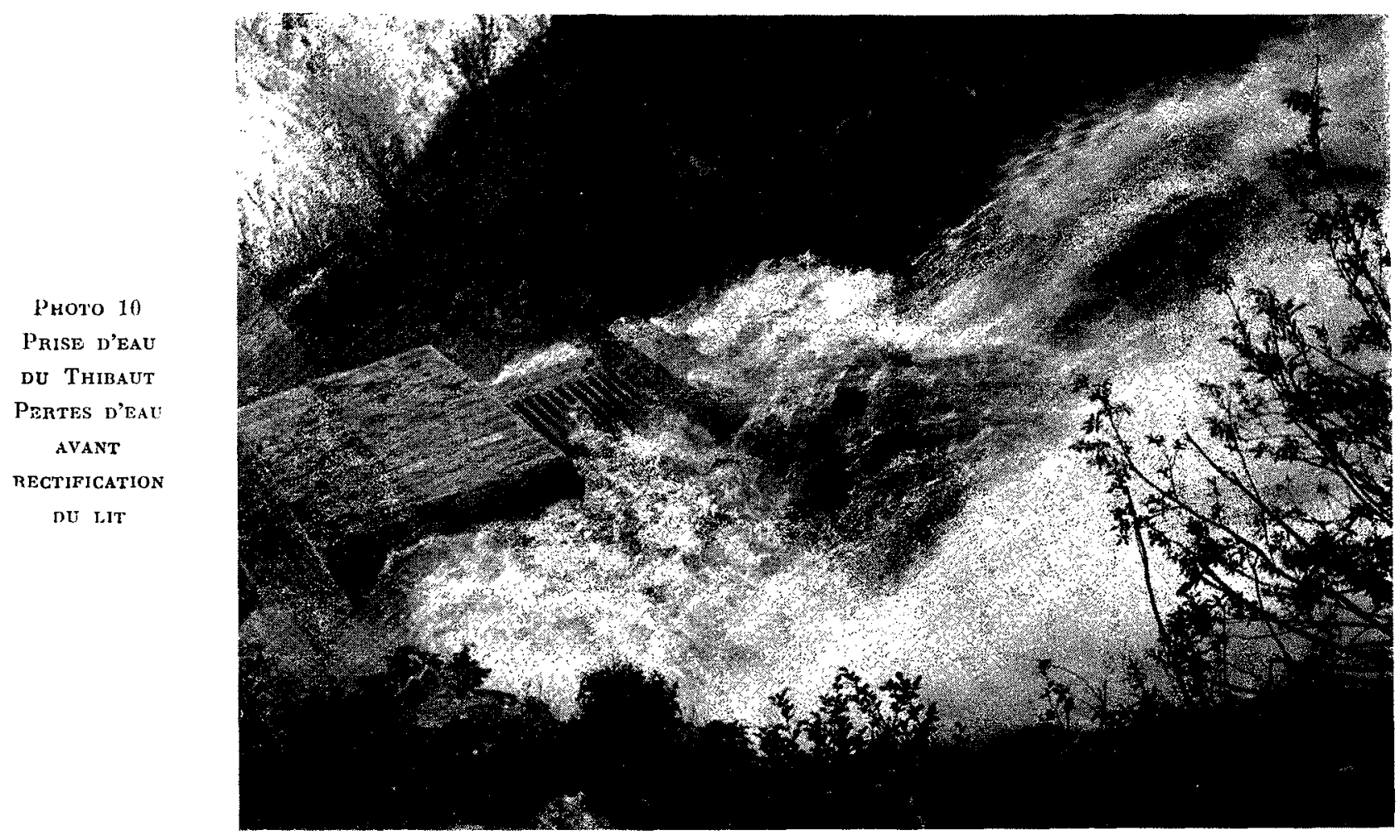

lisées ä la région, ont permis de préciser les caractéristiques d'un profil appelé couteau qui nous parait présenter une grande résistance mécanique et des qualités hydrodynamiques intéressantes. Dans la plupart des cas également, si on a besoin d'une grande solidité, on pourra recourir à des rails inversés; il est, dans ce cas, indispensabie de prévoir, sur le patin, une garniture de forme ronde ou à angle droit qui permet de faciliter l'entrée de l'eau. Les rails, cependant, surtout quand il fait très froid, ne présentent pas toujours les qualités de résilience nécessaires à la résistance aux chocs, par exemple aux avalanches. En tout état de cause, il nous parait indispensab'e qu'ils aient une inertie transversale assez grande sinon les cailloux peuvent les déformer par coïncements et il est ensuite extrêmement difficile de les enlever.
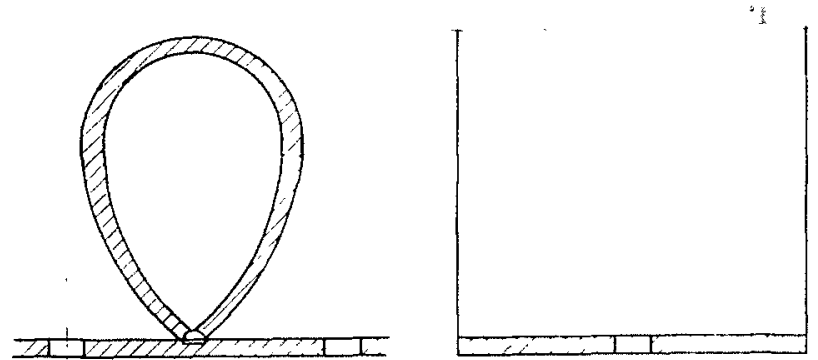

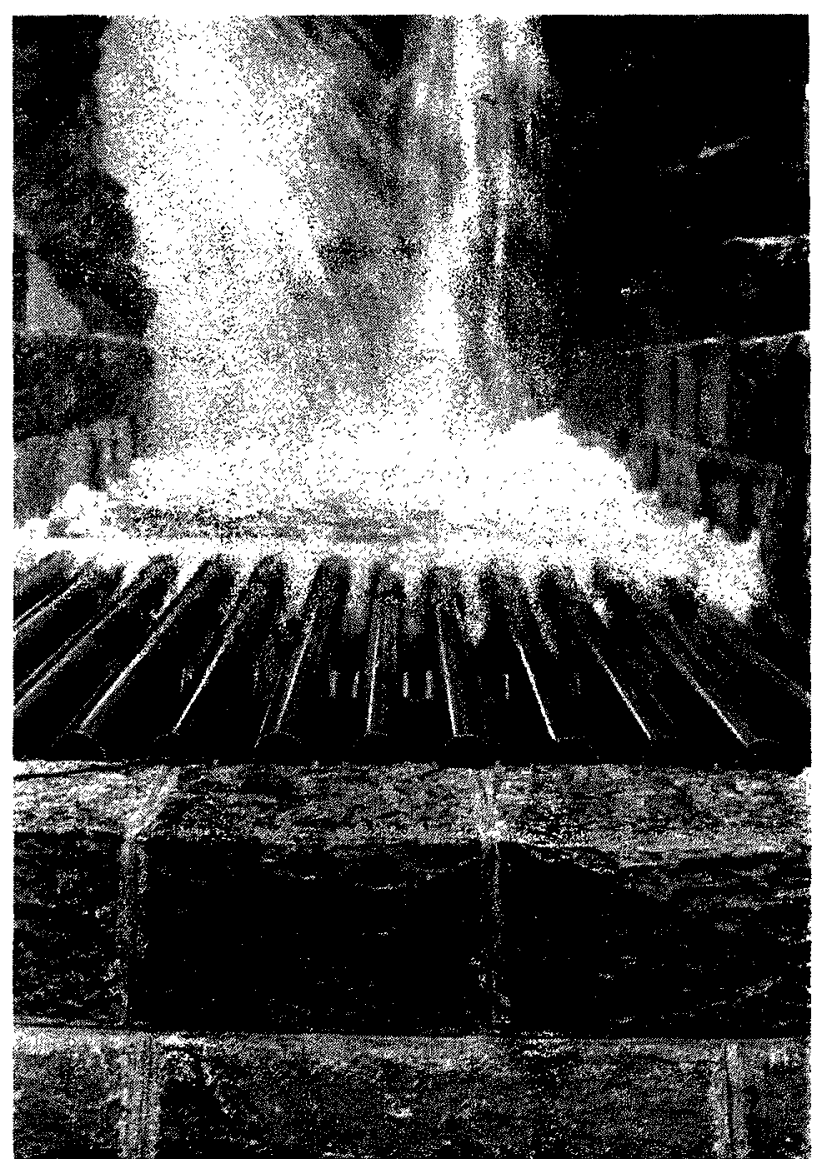

Photo 11. -.. Barreaux de gnille, typj « Coutrau $》$ 


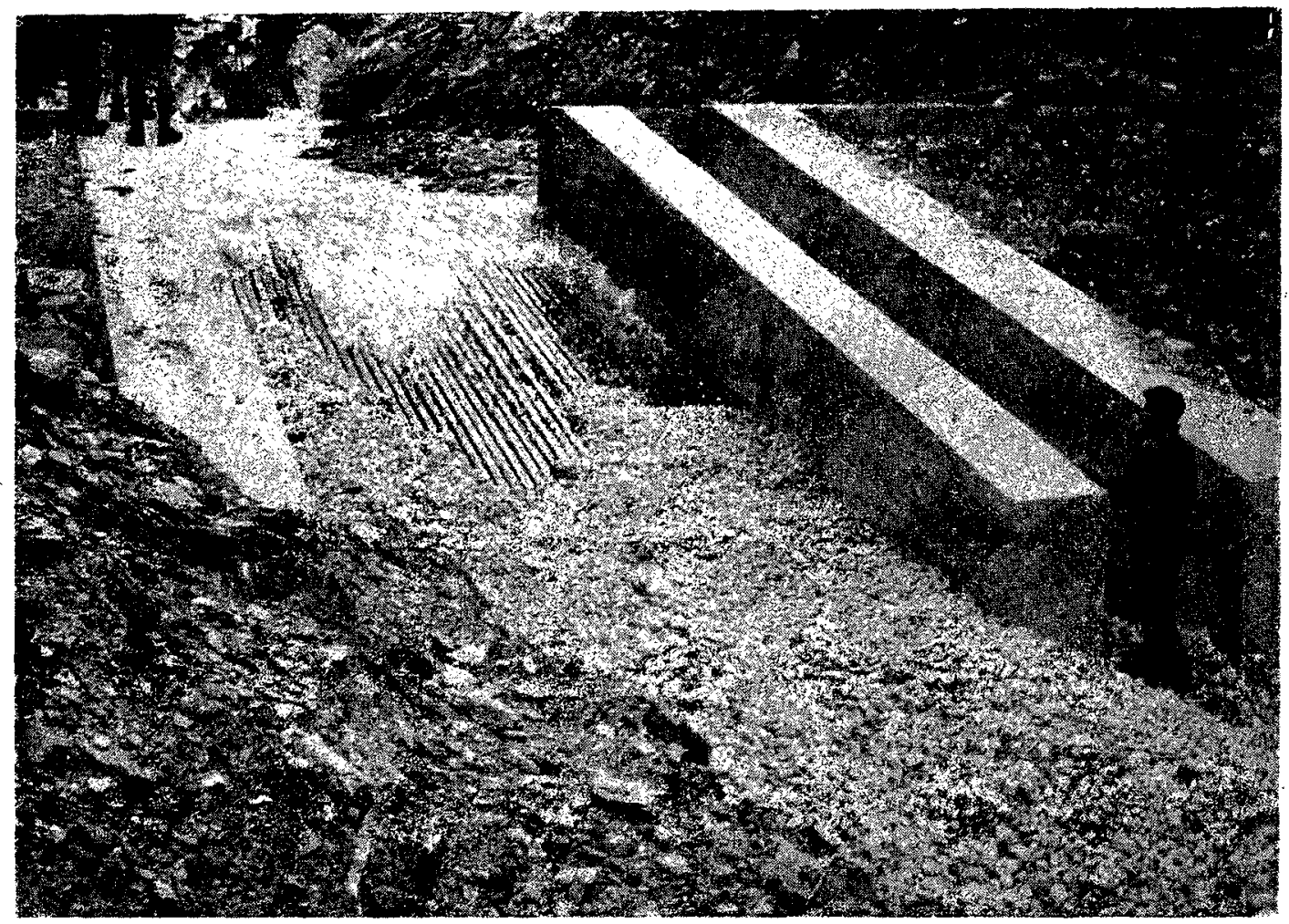

Рното 12

BarreaUX

INSUFFISAMMEN'T

ÉCARTÉs

DËPOTS

DE CAILlOUX

A L'AYAL

DE LA PRISE

Au point de vue pente, il est bon, pensons-nous, de prévoir une valeur de l'ordre de $20 \%$, on pourrait toutefois l'augmenter au cas où des feuilles risqueraient d'obturer les intervalles.

\section{b) Ecartement des barreaux :}

L'expérience d'Aussois nous a conduits à augmenter de plus en plus l'espace entre les barreaux. Au Grand Pyx, au Pingon, et à la Letta, nous avons actuellement laissé en accord avec l'Exploitation des écartements de l'ordre de $10 \mathrm{~cm}$. Le principal avantage de cette disposition, outre qu'elle résoud le problème du gel, réside dans la diminution des risques de bouchage des grilles par les cailloux; en effet, les cailloux de plus de $10 \mathrm{~cm}$ de diamètre sont rares et ne se déplacent véritablement que pendant les crues déjà importantes. En temps normal, tous les transports de fond passent au travers des grilles. Il va sans dire que cette disposition ne marche que si les organes de chasse qui se trouvent à l'aval des grilles sont suffisamment puissants pour entraîner les cailloux les plus gros. Disons, tout de suite, qu'avec le type de dégraveur à purge discontinue que nous avons utilisé à Aussois, la chasse des cailloux les plus gros ne pose aucun problème; nous verrons plus loin qu'avec l'automatisme l'écartement des barreaux s'impose encore plus.

On pourrait nous opposer que cette disposi- lion fait rentrer beaucoup plus de matériaux solides dans la prise d'eau avec, comme conséquence, un supplément du nombre de chasses et des pertes d'eau. En réalité, cet inconvén.ent est mineur car la proportion de gros cailloux est extrêmement faible par rapport aux sables et aux petits graviers qui forment la grosse majorité des apports et qui pénètrent de toute façon dans le dégraveur. En revanche, cette disposition a l'avantage de limiter les dépôts dans le lit à l'aval de la prise, dépôts que les crues n'ont pas la force d'entraîner quand la prise est très suréquipée comme c'est généralement le cas quand on alimente une réserve. Ces dépôts risquent parfois de former à l'aval une véritable colline de cailloux qui peut remonter sur la prise elle-même; il faut alors pratiquement les dégager à la pelle. La photo ci-dessus le prouve surabondamment. Remarquons, en passant, qu'à ce point de vue là, il est bon de ne pas trop enfoncer la prise dans le lit du torrent à moins de dispositions topographiques favorables (forte pente à l'aval).

\section{c) Possibilités DE DÉRIVER LES EAUX A COTÉ DES GRILLES :}

Il est très utile, comme l'a d'ailleurs proposé M. Montagné, de prévoir en parallèle, avec les grilles, une passe plus profonde obturée en exploitation par des batardeaux en bois. Il est 
ainsi facile de mettre les grilles hors circuit pour procéder à leur réparation.

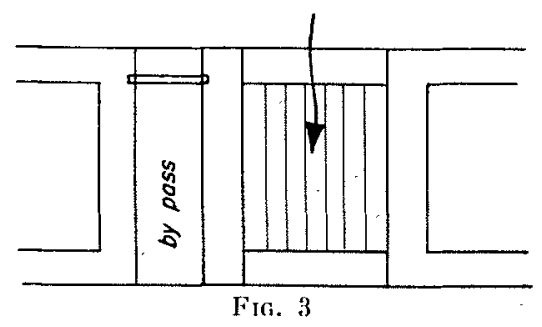

\section{$4^{\circ}$ Limitation des débits à la prise.}

Si les grilles en dessous s'adaptent bien au site et sont particulièrement robustes, elles ont un défaut : c'est de ne limiter pratiquement pas le débit pouvant les traverser. S'il n'y avait pas, à l'aval de ces grilles, un ouvrage destiné à limiter les débits en temps de crue, il faudrait donner aux ouvrages aval des dimensions inadmissibles et coûteuses, ne serait-ce que pour la sécurité du gardien. Cette limitation se fait au Grand Pyx par la vanne à secteur à niveau constant. Ce système paraît, à première vue, intéressant en ce sens que les eaux excédentaires sont prises sous la vanne, là où elles sont le plus chargées de sables, alors que dans d'autres systèmes on déverse les eaux propres de surface. Malheureusement, il n'est pas à l'abri d'un mauvais fonctionnement de la vanne automatique et, de plus, son avantage est plus illusoire que réel parce qu'une vanne fonctionnant en charge ne dégrave pratiquement qu'un cône étroit contre elle-même.

A la prise du Thibaut, au contraire, la limitation se fait au moyen d'un masque conjugué avec un déversoir, le masque étant situé sur le déversoir, lui-même établi à l'aval du premier dégraveur. L'inconvénient de ce système est de ne limiter le débit qu'à l'aval du premier dégraveur qui doit alors être prévu pour pouvoir supporter sans dommage des augmentations de débit considérables.

Pour nos futures prises de Roselend, nous avons pensé à un troisième système qui consiste

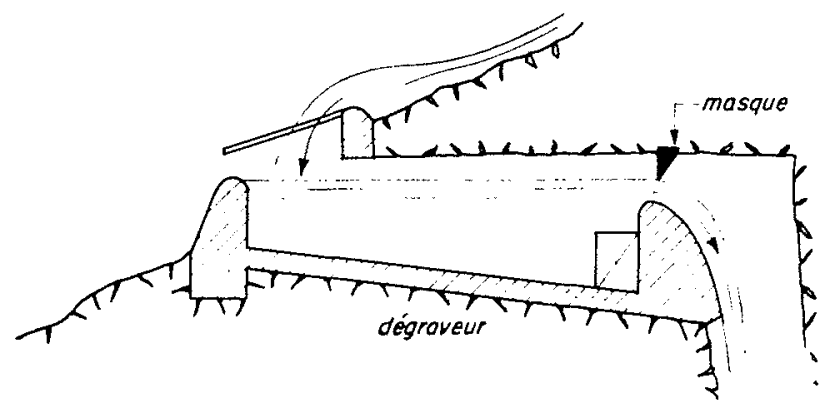

Fig. 4 à limiter les eaux directement sous les grilles par un resserrement, de telle manière que lorsque le débit d'équipement est atteint, la fosse est pleine et les grilles déversent. Ce système a ainsi accessoirement l'avantage de provoquer, en temps de crue, un nettoyage relatif des grilles et des dépôts possibles à l'aval de la prise dans le torrent.

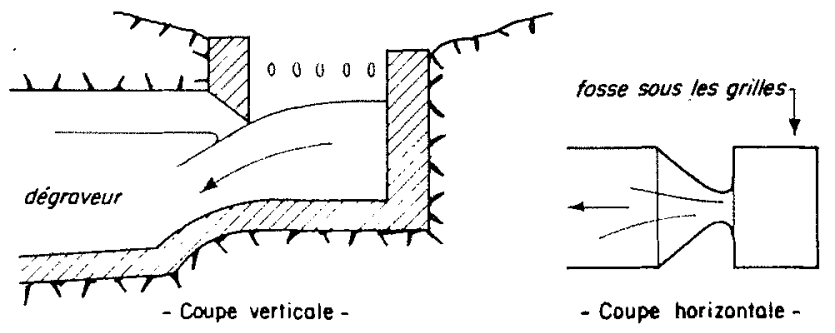

Fig. 5

Tout en permettant une limitation sensible du débit, il ne provoque pas, à l'entrée du dégraveur, une turbulence exagérée qui pourrait être néfaste pour le dépôt des graviers; nous avons d'ailleurs essayé ce système sur modèle réduit à la région. Un autre avantage, qui apparaîtra surtout lorsque nous parlerons des dégraveurs et de l'automatisme, est que, comme il y a une section de contrôle à l'entrée du dégraveur, le niveau dans la fosse est indépendant du niveau du degraveur; autrement dit, que le dégraveur soit en fonctionnement ou en chasse, les eaux dans la fosse se comportent de la même manière - ceci intervient, comme nous le verrons, au sujet de la rapidité des chasses.

\section{$5^{\circ}$ Avantages du système de dégravage ou dessablage à purge discontinue dans le cas de prise de haute montagne.}

Pour se protéger contre les transports solides, il existe en gros deux systèmes de principe assez différent :

- Le système à purge continue qui a besoin d'un débit de fuite pour évacuer les sables et graviers, et dont le plus connu est le dessableur Dufour;

- Le système à purge discontinue constitué par une simple fosse de décantation dans laquelle on fait des chasses périodiques et dont le type pourrait être représenté par le dessableur de $M$. Waeber à la prise d'eau sous-glaciaire de 'Tré-la-Tête.

Pour notre part, nos préférences vont au
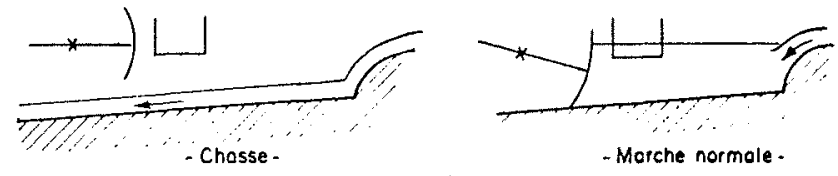

FIG. 6 
deuxième système, tout au moins quand il s'agit de prises de haute montagne. Les avantages que nous lui trouvons sont les suivants :

a) Simplicité : Le génie civil d'un tel ouvrage est particulièrement simple comparé à celui du système à purge continue; c'est un gros avantage pour une prise d'accès difficile où les conditions de travail sont dures.

b) Il est adapté au régime des apports solides : en effet, plus un torrent est petit, plus son régime est variable et ses crues rapides. Ses apports solides arrivent à la prise d'une manière saccadée; on peut avoir pendant 15 jours de l'eau claire et le $16^{\circ}$ une crue de deux heures transportant des dizaines de $\mathrm{m}^{3}$ de cailloux et de sables. Le système à purge discontinue semble mieux adapté à ce régime.

$\mathrm{Au}$ contraire, avec un dessableur à purge continue, en dehors de la période de hautes eaux et pour ne pas perdre trop d'eau, le gardien ferme, en général, le système de purge. Si une crue inopinée survient et que le gardien n'est pas là pour ouvrir les vannes à temps, le dessableur fonctionne en chambre de décantation ordinaire et se bouche. Il faut ensuite - ce qui est très long et nécessite des arrêts d'exploitation - le vider à la pelle, à moins, comme nous l'avons fait par ailleurs, d'avoir prévu une marche éventuelle en discontinu.

c) Il permet donc de limiter le nombre de visites de gardiens.

d) Il est possible de lui adapter un système d'automatisme par horloge hydraulique, donc, dans ce cas, de se passer complètement de gardien.

e) Il économise de l'eau : tant que les eaux sont propres, on ne fait pas de chasse et on ne perd pas d'eau; après une crue, le gardien vient faire une chasse qu'il fait durer le temps juste nécessaire pour nettoyer le bassin de décantation - rendement maximum - perte d'eau minimum.

f) Il permet enfin d'augmenter l'écartement des barreaux de grilles, donc de diminuer leurs risques de bouchage. En effet, si la forme de la fosse est bien étudiée, on peut pratiquement chasser des cailloux allant jusqu'à $20 \mathrm{~cm}$ de diamètre. Un système à purge continue est bien plus délicat et nécessiterait, dans ce cas, une première fosse de dégravage pour se protéger.

\section{$6^{\circ}$ Intérêt d'un calage judicieux de la cote du dessableur par rapport au niveau des grilles en rivière.}

Quand une prise alimente une galerie à écou- lement libre se déversant dans un lac, il lui suffit d'un dégravage sommaire car il est inutile de perdre de l'eau pour éliminer sables fins et limons qui seront surabondamment décantés dans le lac et transiteront sans difficulté dans la galerie. Il faut donc concevoir les prises de telle manière qu'en cas de défaillance du système de purge, la prise ne se bouche pas, mais au contraire fasse transiter les cailloux dans la galerie qui n'en souffrira pas si la circonstance est exceplionnelle. Il n'y a pas de doute que lorsqu'on alimente une réserve, il faut donner priorité à l'eau, la question du dégravage étant, sous les réserves que nous avons indiquées plus haut, relativement secondaire. Pour aboutir au résultat cherché, il suffira de construire une prise relativement haute par rapport au dégraveur, de manière que, si le gardien laisse le bassin de décantation se remplir complètement de graviers par suite d'une négligence, l'eau puisse monter sous les grilles d'une hauteur telle qu'elle aura l'énergie suffisante pour entraîner les cailloux par-dessus le déversoir $D$. Remarquons que, lorsque le gardien reviendra, il fera une chasse qui rétablira sans difficulté la situation comme avant, contrairement à ce qui se passerait avec un dessableur à purge continue.

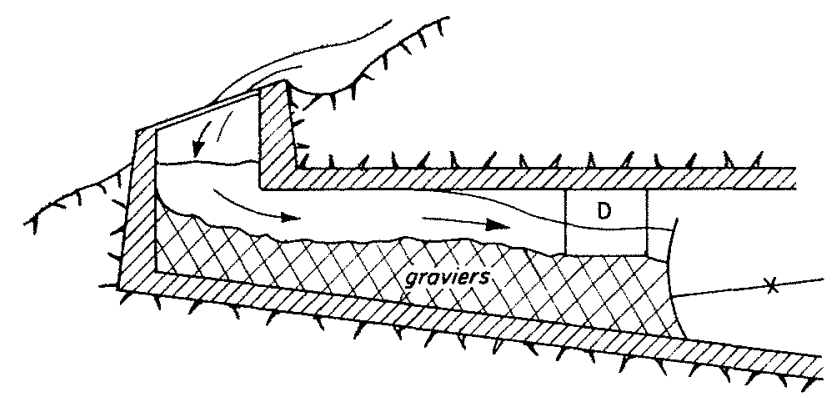

FIG. 7

Au contraire, quand une prise d'eau alimente une galerie dont les eaux sont directement turbinées, il ne faut absolument pas que les cailloux passent en galerie, pour des raisons évidentes. Ainsi, contrairement au cas précédent, il est préférable, en cas de crue catastrophique surprenant le gardien, d'arrêter l'admission d'eau plutôt que de faire entrer des cailloux en galerie. Il suffit, pour obtenir ce résultat, de caler le dessableur très haut par rapport à la prise de manière que lorsqu'il $y$ a excès de cailloux dans le dessableur, la perte de charge qui en résulte fasse remonter le niveau amont au-dessus du niveau des grilles; il y a alors débordement au travers des grilles, diminution de la quantité d'eau admise, ralentissement de la vitesse et dépôt supplémentaire de cailloux; le phénomène se continue ainsi jus- 


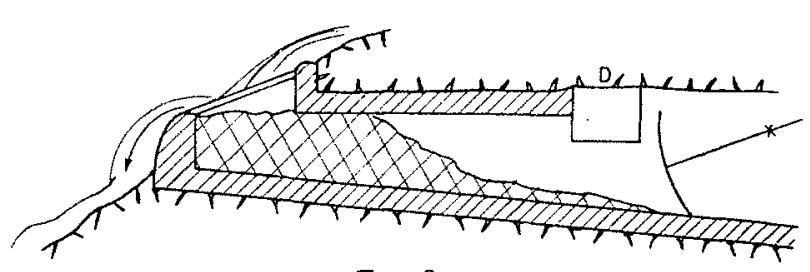

FIG. 8

qu'au bouchage complet de la prise d'eau. Si la prise est judicieusement calculée, ce bouchage doit se produire avant que le talus d'engravement n'arrive au droit du déversoir, donc avant que les cailloux ne transitent en galerie.

\section{$7^{\circ}$ Quand le cas se présente, utilisation de la fenêtre d'attaque comme canal de dé- cantation supplémentaire servant soit à pallier une défaillance du dessa- bleur, soit à faire un dessablage com- plémentaire.}

Il arrive souvent que les travaux nécessitent une fenêtre d'attaque à proximité d'un torrent secondaire que l'on veut capter. Il ne coûte presque rien d'utiliser cette fenêtre, parfois assez longue, comme ouvrage de décantation supplémentaire.

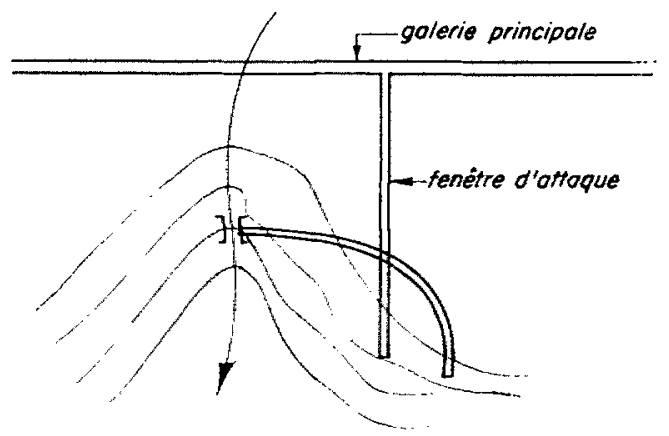

FIG. 9
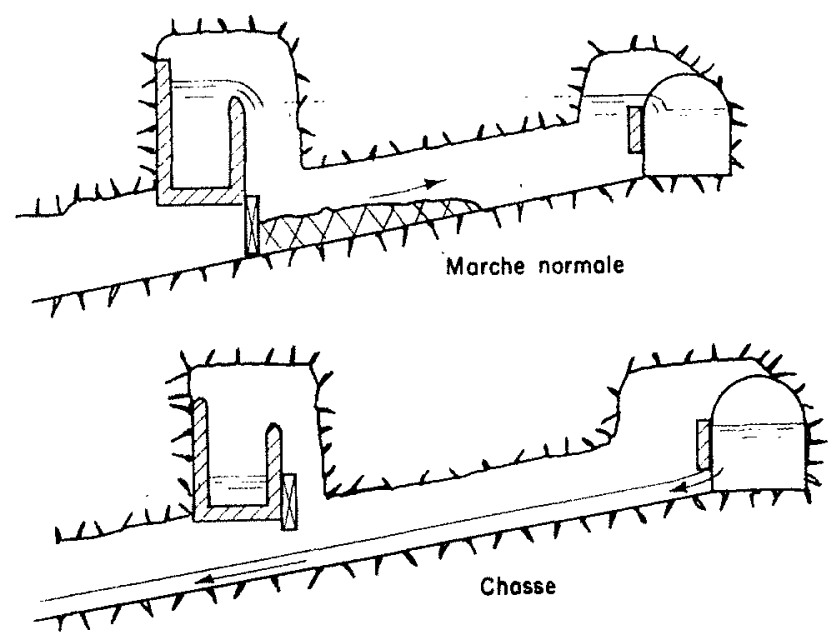

Fig. 10
Une telle fenêtre, par suite de sa longueur imposée, a, en général, un volume utile bien supérieur à celui de la fosse de décantation. Elle peut être utilisée à la fois comme organe de dessablage et, également, comme organe de sécurité, au cas où le dessableur serait complètement engravé.

Les chasses peuvent se faire d'une manière très efficace en utilisant l'eau de la galerie principale. Pour avoir un débit de chasse aussi régulier que possible, nous conseillons de séparer la galerie de la fenêtre par un diaphragme comme indiqué sur le croquis et qui peut être simplement constitué par un batardeau de planches.

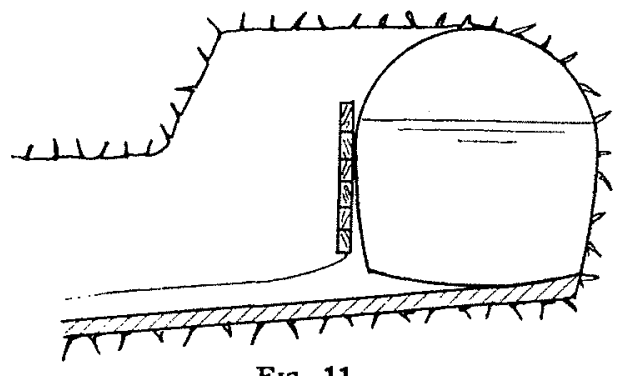

Fia. 11

L'orifice inférieur est calculé pour laisser passer un débit optimum pour une chasse, compte tenu de la largeur de la fenêtre. Si l'orifice est placé suffisamment bas, ce débit de chasse varie beaucoup moins que le débit en galerie et peut être calculé à une valeur optimum, compte tenu des caractéristiques de la fenêtre, de manière à produire une chasse rapide et puissante. Au contraire, dans le système utilisant uniquement l'eau d'une prise secondaire pour la chasse, le gros inconvénient est, en période d'étiage, de ne plus avoir assez de débit, ce qui a pour effet de prolonger la durée des chasses d'une manière considérable. En exploitation normale, l'eau se déverse dans la galerie, à la fois par cet orifice et par le déversoir supérieur (celui-ci étant largement dimensionné); ce déversoir est arasé audessus du niveau maximum des eaux dans la galerie principale afin que le débit de chasse ne provienne que de l'orifice inférieur.

Un tel système a de nombreux avantages; d'abord, il ne coûte pratiquement rien quand la fenêtre existe; ensuite, il permet un excellent dessablage et, grâce ḋ son grand volume, il donne une sécurité supplémentaire au dessablage de tête. Pour cette dernière raison, il est particulièrement intéressant de l'adjoindre à un dessableur muni d'un système automatique, comme il a été fait au Pingon; cela contribue en somme à la suppression complète du gardiennage. 


\section{$8^{\circ}$ Règles à suivre pour améliorer l'efficacité des chasses. - Intérêt des vannes à secteur.}

Les dessableurs à purge discontinue, s'ils sont, à première vue, d'une grande simplicité, exigent cependant, pour leur bon fonctionnement, d'être construits suivant un certain nombre de règles, que nous allons exposer rapidement :

\section{a) SECTION DE CONTROLE A L'AMONT DU CANAL DE DÉCANTATION :}

A l'extrémité amont du canal de décantation, il est bon de prévoir une section de contrôle de manière à séparer hydrauliquement ce canal des organes qui se trouvent à l'amont. De cette manière, on sera sûr, pendant une chasse, de n'avoir à nettoyer que le volume du dessableur et non ce qui se trouve à l'amont; cette remarque est surtout importante dans le cas où le canal de décantation se trouve en rivière. Prenons, par exemple, les deux prises dessinées ci-dessous en coupe : la prise A sans section
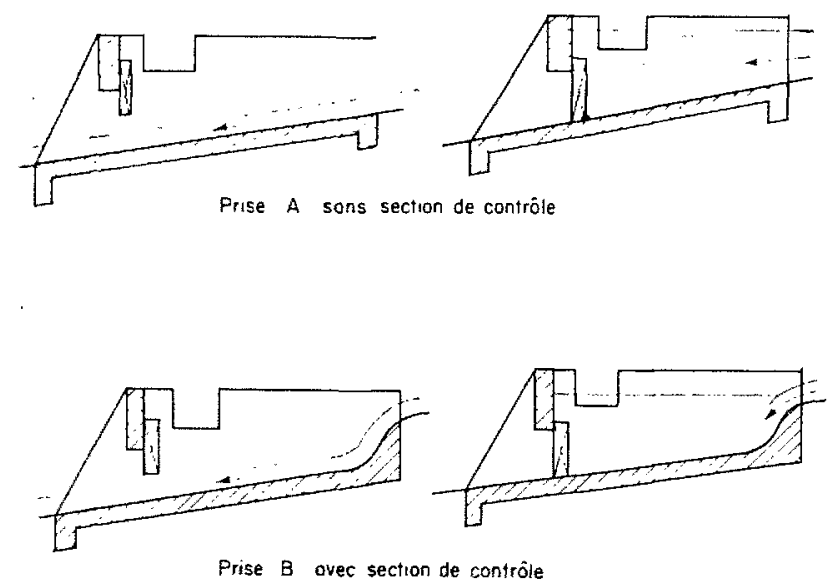

FIG. 12

de contrôle, la prise $B$ avec section de contrôle : quand on fait une chasse en $B$, il n'y a strictement à dessabler (aux apports naturels près) que le volume de sables et graviers stockés dans le dessableur. Une chasse de 5 à 10 minutes suffit en général pour le nettoyer complètement. Dans la prise $A$, on doit dessabler non seulement le canal de décantation, mais encore une partie de la retenue en rivière. L'opération de nettoyage devient alors très longue et, de toute façon, n'est jamais complètement terminée. Les derniers cailloux partent très lentement, presque un par un, et il $\mathrm{y}$ en a souvent un qui se coince sous la vanne de chasse quand on la referme. Le gardien risque alors de détériorer la vanne et, en particulier, de tordre les cré- maillères s'il veut forcer pour la fermer quand même. De toute façon, la durée de la chasse dans la prise $B$ est pratiquement 4 à 5 fois plus courte au moins que dans la prise $A$, ce qui compense largement la diminution de volume de stockage. Dans un torrent à fort charriage, la perte d'eau devient alors sensible.

Dans le cas où le canal de décantation est précédé d'une galerie, il est nécessaire, pour les mêmes raisons, que l'écoulement dans cette galerie soit constamment torrentiel.

\section{b) Canal de nécantation Rectrligne:}

Même lorsque la topographie s'y prête mal, il faut s'efforcer de projeter un canal de décantation rectiligne sur toute sa longueur. Si l'on n'a pas pris cette précaution et si le canal de décantation comprend un coude, au moment d'une chasse, il y a formation d'un tourbillon à l'intérieur du coude juste à son aval, et dépôt de graviers et de sables à cet endroit là. Le fait en lui-même n'aurait pas d'importance, puisque le volume de graviers est absolument négligeable, si les cailloux ainsi stockés ne se trouvaient pas chassés un par un d'une manière tout à fait imprévisible. Comme dans le cas précédent de la prise $A$, il arrive fréquemment an moment où l'on referme la vanne que ces cailloux se coïncent sous elle, d'où les inconvénients dont nous avons déjà parlé.

\section{c) Section du canal et largeur de la VANNE :}

Nous conseillons la forme trapézoïdale indiquée sur le croquis ci-dessous: la dimension importante à calculer est la largeur qui doit être telle que, même en basses eaux, la vitesse de l'eau, au cours d'une chasse, soit suffisante pour entraîner les cailloux les plus gros qui passent

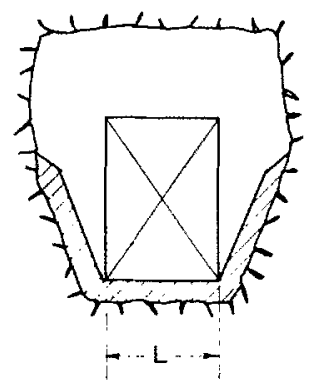

Fig. 13

au travers des grilles. On conçoit assez bien que si L. est trop grand, on risque de ne pas pouvoir dégraver si après une crue le débit du torrent a brusquement baissé.

Une règle impérative, à notre avis, est que la 
vanne de chasse ait pour largeur L; en effet, si sa largeur est inférieure à $L$, elle risque en temps de chasse, de provoquer un ralentissement de l'eau à sa hauteur, donc une augmentation de la durée des chasses. Ce ralentissement a aussi pour conséquence d'arrêter les cailloux juste au droit de la vanne, d'où difficulté pour la refermer.

\section{d) Pente du canal de Décantation :}

Plus la pente est forte, plus le nettoyage est efficace et rapide. Malheureusement, avec une pente de 10 à $20 \%$, on est conduit, à l'aval du canal de chasse, à prévoir des ouvrages d'une hauteur prohibitive, ce qui fait que, pratiquement, nous nous cantonnons dans des pentes de 2 à $5 \%$ qui, — l'expérience le prouve _-, sont bien suffisantes et permettent de nettoyer complètement le canal de décantation en des temps de l'ordre de 10 minutes environ. Une solution intéressante, cependant, consiste à prévoir un dessableur en charge comme indiqué sur le croquis ci-après.

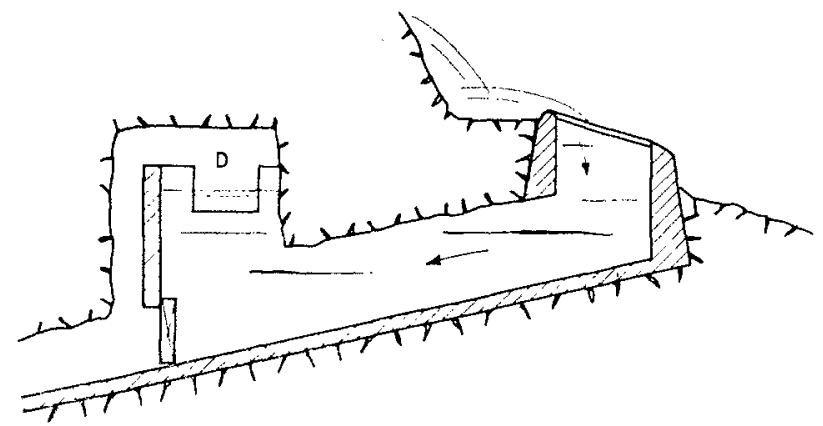

FIG. 14

L'inconvénient de la forte pente ne se répercute alors strictement que sur les ouvrages aval et la section du canal est constante, ce qui est avantageux à la fois pour les travaux et pour la décantation qui se fait alors à vitesse constante. Notons d'ailleurs à ce propos qu'un canal de décantation à écoulement libre et à forte pente en radier se conduit comme un divergent. Plus ce divergent est grand, plus il est difficile d'avoir un régime tranquille. L'eau conserve une certaine turbulence et la décantation des éléments fins devient difficile. C'est une raison de plus, à notre avis, pour ne pas dépasser $5 \%$ de pente dans un dégraveur non en charge.

\section{e) Avantages des vannes a secteur :}

Signalons enfin que chaque fois que nous le pouvons, nous utilisons des vannes à secteur équilibrées; elles sont simples, robustes, faciles à manier, et ont l'énorme avantage de ne pas nécessiter de rainures dans la passe de chasse, ce qui diminue considérablement les risques de coincement. En outre, il est facile de leur adapler, comme nous le verrons plus loin, un système d'automatisme.

\section{$9^{\circ}$ Intérêt de l'automatisme par horloge hydraulique.}

\section{a) Principe du systême:}

Ce système comprend 2 fosses de volume utile égal A et B reliées par un siphon. La fosse A se remplit lentement; quand elle est pleine, le siphon s'amorce et la vide entièrement dans $B$ provoquant la montée complète de la vanne à secteur et une chasse; la vanne d'évacuation C permet de régler la durée de cette chasse.

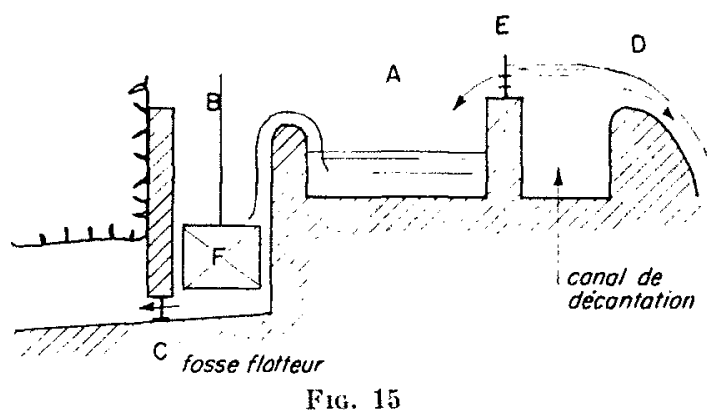

Remplissage de la fosse $A$ : le niveau dans le canal de décantation est réglé par le déversoir $D$. La fosse A est en communication avec ce canal par un jeu d'orifices calibrés $E$. Le volume de $A$ et la disposition des orifices $\mathrm{E}$ permettent de choisir une loi réglant le nombre de chasses en fonction du débit.

Ce système a été entièrement mis au point à la région. Il a fonctionné pendant deux saisons au Pingon où il a donné à l'Exploitation toute satisfaction. Le Pingon est un torrent d'origine glaciaire qui, par conséquent, transite une assez grande quantité de matériaux solides. L'horloge hydraulique est réglée pour faire, pendant les hautes eaux, deux à trois chasses par jour pendant une durée de 12 minutes chacune, ce qui représente une perte d'eau maximum de $2,5 \%$ bien inférieure aux $10 \%$ généralement admis dans un dessableur à purge continue. D'autre part, dès que le débit baisse, la cadence de chasse diminue automatiquement jusqu'à une chasse par semaine, ce qui représente une perte d'eau de $0,12 \%$ absolument négligeable. Pendant deux ans, cette prise d'eau a été simplement visitée par le gardien qui n'a eu à s'assurer que de sa bonne marche; aucune panne n'a été constatée et la vanne n'a jamais été coüncée 
par les cailloux, ce qui prouve que chaque fois qu'elle se referme après une chasse, le canal de décantation est complètement nettoyé. Une prise d'eau de ce type, si elle est bien réglée, permet donc de se passer presque complètement de gardien. Dans un aménagement ayant un grand nombre de petites prises d'eau conme à Aussois, un seul gardien suffirait pour toutes les prises munies d'automatisme; son rôle consisterait simplement à faire des rondes périodiques pour s'assurer de la bonne marche des systèmes.

b) Précautions a prendre pour ob'eNIR UN FONCTIONNEMENT SUR ET EFFICACE :

Une prise d'eau qui marche bien doit cette particularité à une foule de petits points de détail qui peuvent paraître secondaires à première vue, mais qui, lorsqu'ils sont négligẻs, risquent de perturber gravement la bonne marche d'un ensemble pourtant bien étudié. Quand la prise est gardiennée, il est toujours possible de remédier à ces insuffisances par un travail supplémentaire du gardien. Au contraire, quand la prise est automatique, la moindre défaillance peut provoquer des dégâts importants puisqu'il n'y a personne pour y remédier rapidement. Il est donc particulièrement indispensable dans ce cas de multiplier les précautions pour obtenir une marche absolument impeccable.

C'est sous cet angle que nous allons passer en revue les organes des adductions automatiques dont le bon fonctionnement nous semble absolument indispensable pour la bonne marche de l'ensemble.

- Grilles : Il est inutile de faire une prise automatique si les grilles en rivière se bouchent, se cassent, ou même font simplement perdre de l'eau à la prise.

Dans nos régions, les grilles ne risquent, en général, pas de se boucher à cause des feuilles. Notons d'ailleurs, à ce point de vue là, que les prises en dessous se bouchent très difficilement lorsqu'elles ont une pente supérieure à $30^{\circ}$. Au contraire, le risque de bouchage par les cailloux est grand quelle que soit la pente. Comme on l'a vu, la manière la plus radicale de résoudre la question est d'écarter les barreaux en laissant entre eux des intervalles de 10 a $15 \mathrm{~cm}$.

Une sécurité supplémentaire consiste à prévoir une surface de grilles très surabondante de manière que, même quand elles sont partiellement bouchées, la surface libre restante soit encore suffisante pour laisser passer le débit d'équinement. Notons en passant qu'il est indispensable dans ce cas de prévoir un organe de limitation de débit suffisamment efficace à l'aval immédiat des grilles. Il est bon de prévoir, également, sur les grilles une passerelle sommaire, si besoin amovible, permettant au gardien de nettoyer les grilles en cours d'exploitation sans être dans une position acrobatique et sans trop se mouiller.

- Durée des chasses: Quand toutes les précautions nécessaires ont été prises de telle sorte que les chasses n'intéressent strictement que le bassin de décantation, la durée maximum d'une chasse peut alors être facilement trouvée expérimentalement car elle est égale ả la durée d'une chasse faite en période de basses eaux, le dégraveur étant complètement engravé. Une telle mesure est indispensable pour le réglage de la durée d'ouverture de la vanne à secteur. O'n sera sûr, de cette manière, que lorsque la vanne de chasse se refermera, le canal de décantation sera complètement nettoyé et qu'il n'y aura aucun risque de coïncement de cailloux sous la vanne et par conséquent pas de perte d'eau.

Il est bon cependant de remarquer que lorsque la prise est automatique, la fréquence des chasses augmente avec le débit du torrent et qu'il y a de fortes chances pour que la chasse se fasse en réalité avec un débit voisin de celui qui a transporté les cailloux dans le torrent, donc, en général, avec un débit assez fort. La chasse n'en est que plus rapide et la sécurité de bon fonctionnement plus grande.

- Horloge hydrauliane; réglage de la fréquence des chasses : L'horloge hydraulique est l'organe qui fait varier la fréquence des chasses selon le débit rentrant dans la prise (voir croquis). Cet appareil est constitué par une boîte métallique en communication avec le canal de décantation par un tuyau et qui peut être bypassé par une vannette quand on veut ouvriy volontairement la vanne de chasse. Cette boîte

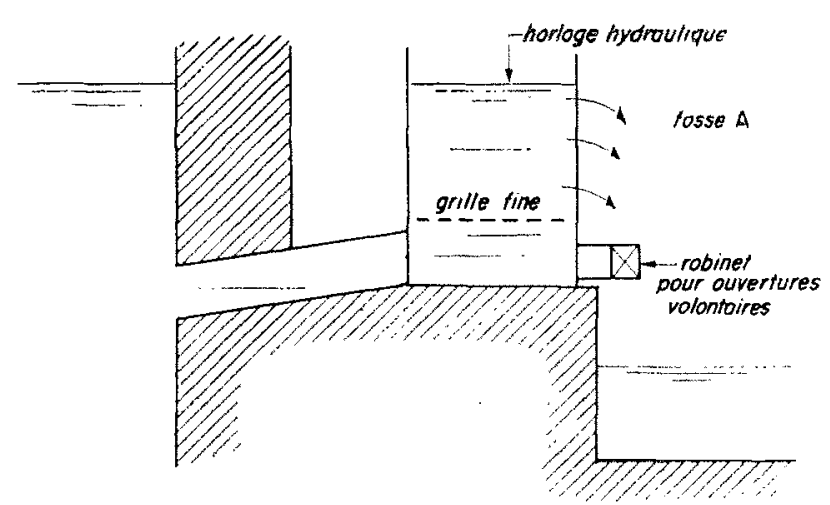

FiG. 16

métallique est percée d'un certain nombre de trous calibrés dont le nombre et la dimension servent à reproduire la loi : fréquence des chasses-débit, que l'on s'est donnéc. Notons qu'il 


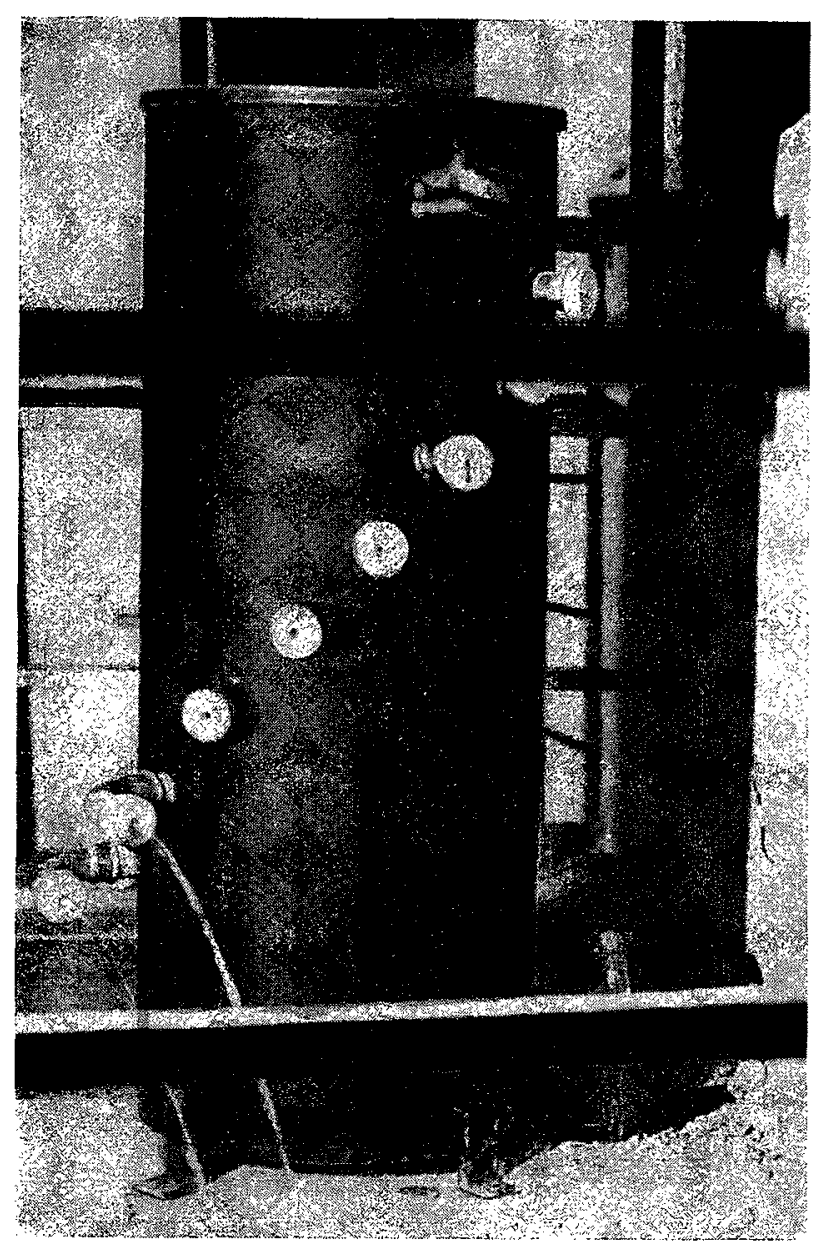

Photo 13. - Horloge hydraulique

faut prendre certaines précautions pour que les orifices en question ne se bouchent en aucun cas. Pour cela, il suffit de prévoir une grille fine dans le fond de l'horloge hydraulique; cette grille sera d'ailleurs automatiquement nettoyée pendant les chasses par le courant de retour provoqué par la baisse du niveau d'eau dans le canal de décantation.

- Ouverture volontaire de la vanne : Le système d'automatisme permet l'ouverture hydraulique volontaire de la vanne, même à l'étiage. En effet, il est souvent difficile d'ouvrir volontairement une vanne à secteur équilibrée avec flotteur parce que, à partir du moment où l'on admet l'eau dans le puits du flotteur, la vanne commence à se lever. Le dégraveur se vide et le niveau devient trop bas pour qu'on puisse continuer à alimenter le puits du flotteur. La vanne s'arrête, en général, avec une très faible levée. Au contraire. avec le système dont nous venons de parler, il est facile de remédier à cet inconvénient en prévoyant une vannette courcircuitant l'horloge hydraulique. Quand on veut ouvrir la vanne, on ouvre la vannette de courcircuitage qui remplit rapidement la fosse A sans que la vanne de chasse ne bouge. Quand la fosse A est pleine, le siphon se déclenche et remplit la fosse $B$ levant la vanne secteur au maximum.

- Siphon et vitesse d'ouverture de la vanne de chasse : Il est nécessaire d'utiliser un siphon qui se deelenche même avec un très faible débit d'amorçage. Nous avons trouvé le problème complètement résolu en utilisant les appareils automatiques de chasse d'eau pour égouts qui s'amorcent, grâce à un système pneumatique, non pas avec un débit d'amorçage, mais à partir d'une certaine charge d'eau. Ces appareils sont très robustes et ne nécessitent aucun entretien. La vitesse d'ouverture de la vanne secteur de chasse dépend du débit de ce siphon; si ce débit est trop fort, la vanne se lève très rapidement, provoquant dans le torrent une crue intempestive heureusement très courte. Il est bon, en conséquence, de calculer le débit du siphon pour que la vitesse de levée de la vanne de chasse soit telle que le débit de chasse, qu'elle laisse échapper pendant le temps où elle fonctionne encore en charge, n'excède pas le débit d'apport du torrent dans une proportion exagérée.

- Flotfeur de la vanne à secteur : La plupart des ennuis que l'on a avec les vannes à secteur équilibrées viennent de ce que le flotteur n'est, en général, pas suffisamment dimensionné pour vaincre les frottements parasites que le projeteur n'avait pas prévus dans ses calculs, en particulier, le frottement des cailloux contre le tablier de la vanne lorsque le canal de décantation est engravé. Un moyen simple et efficace de parer à l'éventualité d'une telle panne est de multiplier carrément par deux le volume du flotteur projeté par le constructeur car, à part le prix, il n'y a, à notre avis, aucun inconvénient technique à augmenter son volume.

- Orifices de vidange du puits du flotteur. Réglage de la durée des chasses : La vannette de chasse $\mathrm{C}$ du puits du flotteur commande le temps de vidange de ce puits, done la durée des chasses. Nous avons indiqué plus haut la manière dont cette durée peut être calculée. Notons, en outre, deux points de détail qui ont leur importance :

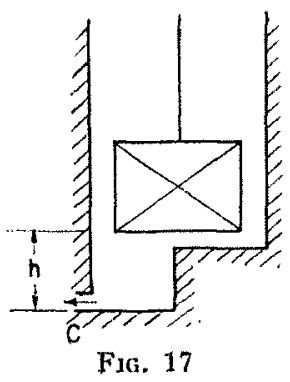


Celte vannette ne doit pas être calée trop près du flotteur; il est bon de la caler au contraire à une distance $h=0,50 \mathrm{~m}$ à $1 \mathrm{~m}$ sous le fond du flotteur; ceci pour que le flotteur ait encore une vitesse appréciable au moment de la fermeture de la vanne. En effet, si celle-ci se ferme trop lentement, son étanchéité risque d'être moins bonne.

L'expérience du Pingon nous a montré également qu'il était bon de doubler cette vannette d'un orifice calibré circulaire servant à régler la durée des vidanges de la vanne de chasse, la vannette servant uniquement au nettoyage de la fosse du flolteur.

\section{$10^{\circ}$ Confort du gardien, accès.}

Pour terminer, nous pensons qu'il est bon de dire quelques mots au sujet du confort du gardien de prise d'eau. En effet, cet aspect de la question est, en général, complètement oublié par le projeteur qui trouve astucieux de faire des économies en faisant des accès acrobatiques et même dangereux et des chambres de vannes minuscules, particulièrement inconfortables et traversées, en général, par des courants d'air mortels. Il suffit souvent de quelques petites précautions prévues dès le début, et d'ailleurs peu conteuses comparées au prix total de la prise, pour changer complètement l'aspect et les facilités d'exploitation d'une prise d'eau.

Signalons, en particulier, qu'il est bon :

- De prévoir, dans les endroits dangereux, des rembardes solides;

- De boucher tous les orifices capables de provoquer des courants d'air; ceux-ci, en effet, outre qu'ils sont particulièrement désagréables pour le gardien et les visiteurs, peuvent, pendant certaines périodes, augmenter notablement les risques de gel à l'intérieur de la prise;

- De prévoir, si possible, dans la prise d'eau, une petite chambre de veille avec un lit et une table;

- A défaut, une armoire fermée à clé contenant un outillage complet, une lampe, un imperméable, etc.;

- De prévoir une fosse à machefer en béton armé située à proximité de la vanne à étancher;

- Enfin, de ne pas oublier un accès aux grilles en rivière et de prévoir la possibilité de les nettoyer sans avoir à dériver les eaux par côté.

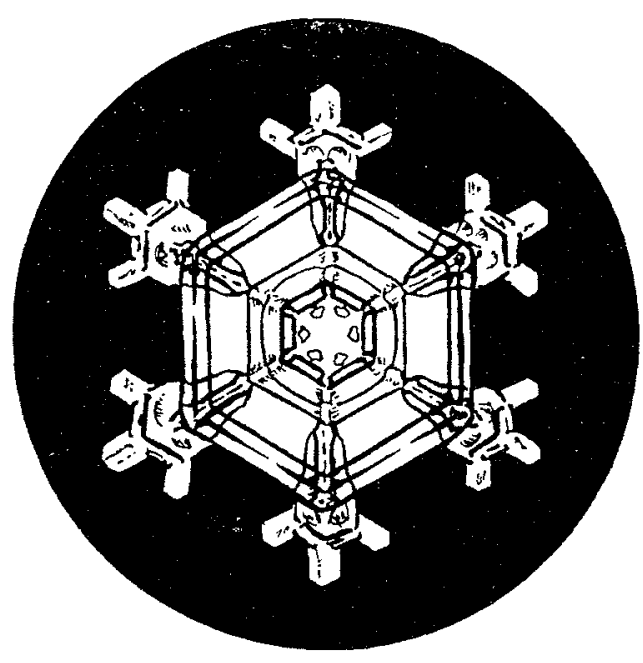

